\title{
Efectos de ciclo vital, generación y periodo en la práctica deportiva de los españoles (1984-2014) \\ Life Phase, Generation and Period Effects on Sports Participation \\ in Spain (19842014)
}

Ramón Llopis-Goig

\section{Palabras clave}

Edad

- Efecto de ciclo vital

- Efecto generación

- Efecto periodo

- Práctica deportiva

- Tiempo

\section{Key words}

Age

- Life Phase Effect

- Generational Effect

- Period Effect

- Sports Practice

- Time

\begin{abstract}
Resumen
Esta investigación presenta un análisis de la influencia de los efectos generacionales, de ciclo vital y de periodo en la evolución de la práctica deportiva de la población española. El análisis se ha referido al periodo 1984-2014 y se ha llevado a cabo mediante la aplicación de un diseño APC (age, period and cohort). El estudio ha constatado el impacto del efecto de ciclo vital sobre la práctica deportiva y la existencia de fuertes efectos de periodo en tres tramos concretos, así como la mayor intensidad de estos dos efectos frente a los de carácter generacional. Los análisis efectuados también han evidenciado que los efectos de periodo han sido los más influyentes en la evolución de la práctica deportiva entre 1984 y 2014.
\end{abstract}

\section{Abstract}

This article presents an analysis of the influence of generational, life phase and period effects on the evolution of sports participation in Spain. The analysis refers to the period between 1984 and 2014 and it was conducted using an APC (age, period and cohort) design application. This study verifies the impact of the life phase effect on sports practice and the existence of strong periodical effects at three specific moments, as well as the greater intensity of these two effects as compared to the generational effects. The analysis reveals that period effects have had the greatest influence on the evolution of sports participation between 1984 and 2014.

\section{Cómo citar}

Llopis-Goig, Ramón (2017). «Efectos de ciclo vital, generación y periodo en la práctica deportiva de los españoles (1984-2014)». Revista Española de Investigaciones Sociológicas, 157: 85-102. (http://dx.doi.org/10.5477/cis/reis.157.85)

La versión en inglés de este artículo puede consultarse en http://reis.cis.es 


\section{INTRODUCCIÓN ${ }^{1}$}

En el curso de las últimas décadas la participación deportiva ha experimentado un notable incremento en la mayoría de países europeos (Scheerder et al., 2011). También en España, donde los estudios realizados muestran que la proporción de personas que realizan una actividad deportiva ha pasado del 12\% de 1968 (Martínez del Castillo et al., 1992) al 47\% de 2014 (Llopis-Goig, 2016). Un aspecto fundamental de ese aumento tiene que ver con la incorporación de las personas mayores a la práctica deportiva. En España, por ejemplo, aunque la relación entre edad y práctica deportiva sigue siendo lineal y negativa (García-Ferrando y LlopisGoig, 2011), el cambio ha sido notable. En 2014, los grupos de 55 a 64 y de 65 y más años registraban unas tasas del 33 y del $17 \%$ respectivamente, cuando tres décadas antes el primer grupo alcanzaba un $4 \%$ y el segundo era ajeno a esta práctica (Llopis-Goig, 2016). En otros países europeos, sin embargo, la tendencia ha sido mucho más intensa y el aumento de la edad ha dejado de implicar una caída de la práctica deportiva (Breedveld y Hoekman, 2011; Gratton, Rowe y Veal, 2011).

Las conclusiones a las que se acaba de hacer alusión proceden en su mayoría de estudios transversales (cross-sectional) cuya principal debilidad consiste en que no permiten especificar si las diferencias relativas a la edad han sido causadas por efectos generacionales o por efectos del ciclo vital. Para identificar los cambios que se producen en el tiempo a medida que los individuos atraviesan diferentes etapas de su vida se requiere el uso de diseños de carácter longitudinal (panel), en los que una misma muestra

\footnotetext{
1 La investigación que se presenta en este artículo fue realizada merced a la concesión de una Ayuda a la Investigación (modalidad $\mathrm{A}$ ) en la Convocatoria de Subvenciones a la Investigación de 2014 del Centro de Investigaciones Sociológicas.
}

es entrevistada sucesivas veces a lo largo de un amplio periodo de tiempo. Los datos procedentes de estudios con diseños de investigación longitudinal permiten el análisis de cambios intraindividuales y -en su aplicación al estudio de la participación deportiva - harían posible la obtención de información sobre la historia específica de abandonos y entradas/reentradas de cada individuo en el sistema deportivo. Tales datos, sin embargo, no se encuentran disponibles en España ni en la mayor parte de los países europeos, aunque pueden realizarse aproximaciones mediante la fusión de diversas encuestas transversales, lo que se conoce como un «falso panel».

Esta investigación se propuso efectuar un análisis del desarrollo de la participación deportiva durante un periodo temporal de treinta años (1984-2014) mediante un diseño APC (age, period and cohort) o análisis de cohortes (Yang, 2011: 17). El objetivo central fue identificar el impacto de los efectos generacionales, de ciclo vital y de periodo sobre la evolución de la práctica deportiva de la población española. Se pretendía contestar tres interrogantes: ¿las diferencias en las pautas de participación deportiva relacionadas con la edad tienen que ver con las situaciones que atraviesa cada persona a lo largo de su vida?, ¿las pautas de cada cohorte pueden explicarse por la influencia de unas circunstancias sociales e históricas que caracterizan el proceso de socialización y desarrollo de cada persona?, y ¿existe algún tipo de influencias globales que hayan determinado la evolución de la práctica deportiva de la población española en los últimos treinta años?

El trabajo contiene un primer apartado en el que se presentan las características del modelo APC, el planteamiento teórico y las hipótesis del estudio. En el siguiente se concreta el diseño y las características técnicas de la investigación. A continuación se presentan los resultados más relevantes y, por último, las conclusiones. 


\section{Planteamiento}

\section{EI modelo APC}

A diferencia de lo que suele ser habitual en las investigaciones de carácter transversal -en las que se examina la relación de una determinada práctica o comportamiento con factores de tipo individual (sexo, nivel de estudios, situación laboral o estado civil) - , los estudios englobados bajo la denominación APC examinan el origen y los procesos de cambio social a partir de la edad (efecto de ciclo vital), la cohorte (efecto generacional) y el año de referencia (efecto de periodo), lo que permite identificar el modo en que evolucionan los niveles agregados de un determinado comportamiento en la sociedad.

El estudio de estos efectos en el campo de la práctica deportiva ha sido escaso a nivel internacional e inexistente en España. Los estudios retrospectivos de Engel y Nagel (2011) y Klostermann y Nagel (2014) sobre la práctica deportiva a lo largo del ciclo vital han revelado la existencia de una tendencia caracterizada por progresivos abandonos y entradas/reentradas a lo largo de la vida de cada individuo, que pueden ser considerados como resultado de los cambios que se producen en sus necesidades, demandas y deseos de participación a medida que accede a nuevas etapas. Otra aportación sobresaliente es la de Breuer y Wicker (2009), en la que se combina el análisis longitudinal con el análisis de cohortes con el objeto de validar las diferencias de edad detectadas en estudios previos de carácter transversal. En su análisis - realizado también, como el de Engel y Nagel (2011), en Alemania- detectaron que los efectos de cohorte eran los que suponían una mayor contribución al desarrollo de la práctica deportiva, y pese al paso del tiempo lograban mantener constante e incluso incrementar - a pesar de los efectos derivados del aumento de la edad- el nivel de práctica deportiva dentro de cada cohorte. Breuer y Wicker (2009) también se refirieron a los efectos de periodo, que explicarían la creciente importancia de la salud y habrían modificado las normas sociales referidas a la edad dejando atrás la imagen de la tercera edad como una etapa de inactividad física. De este modo, los efectos de periodo estarían compensando -e incluso superandolos efectos de ciclo vital (edad) identificados en las encuestas transversales. Otro trabajo realizado desde el modelo de análisis APC es el de Maja Pilgaard (2013), en el que se examinó la evolución de la participación deportiva en Dinamarca. Los resultados obtenidos por Pilgaard ponen de manifiesto que la participación deportiva de los daneses durante el periodo 1975-2007 se ha visto afectada por los tres tipos de efectos, siendo los de periodo los de mayor impacto, seguidos de los de ciclo vital. La investigación que se presenta en este trabajo se sitúa en la senda abierta por las investigaciones de Engel y Nagel (2011), Klostermann y Nagel (2014), Breuer (2005), Breuer y Wicker (2009) y Pilgaard (2013), especialmente de esta última, de la que adopta sus hipótesis y estrategia de análisis para ponerlas a prueba en la sociedad española.

\section{Marco teórico e hipótesis}

La hipótesis del efecto generacional - también llamado efecto cohorte- parte de la idea de que el contexto deportivo en que se socializa cada generación afecta a sus creencias y pautas comportamentales posteriores (McPherson, Curtis y Loy, 1993: 37; Coakley, 2015: 52). Las cohortes generacionales consideradas en esta investigación han sido socializadas prácticamente a lo largo de todo el siglo XX, por lo que han disfrutado de distintas posibilidades de acceso a la práctica deportiva.

El primer grupo generacional considerado es el de la generación de posguerra, denominación que incluye a las personas que nacieron en los años veinte y se socializaron en una sociedad subdesarrollada en la que no existían instalaciones deportivas. La co- 
nocida como generación del desarrollo es la de las personas nacidas en los años treinta y cuarenta que vivieron los efectos del Plan de Estabilización de 1959 y los dos primeros planes de desarrollo. La radio les permitió vivir gestas como la derrota que la selección española de fútbol infligió a la inglesa en el Mundial de 1950, la victoria de Bahamontes en el Tour de Francia de 1959 o las cinco victorias consecutivas del Real Madrid en las cinco primeras ediciones de la Copa de Europa de fútbol. La práctica deportiva, sin embargo, seguía siendo muy escasa en un país que carecía de las más básicas infraestructuras deportivas. La etiqueta de generación de la transición se suele atribuir a las personas nacidas en los años cincuenta, socializadas en una etapa en que el país había comenzado a salir del aislamiento y a integrarse en la órbita occidental. El deporte se había convertido ya en uno de los contenidos centrales de los medios de comunicación aunque la práctica deportiva seguía siendo muy escasa. Las personas nacidas en los años sesenta, conocidas como la generación del baby boom, se desarrollaron en un contexto de transición de la dictadura a la democracia y de tránsito a un modelo cultural que supuso el auge de la televisión y una mayor diversificación cultural. La denominación generación $X$ incluye a las personas nacidas en los setenta y socializadas en un contexto marcado por la caída del Muro de Berlín, la integración europea, el avance de los procesos de globalización y el auge de un nuevo paradigma cultural electrónico y digital. A continuación estaría la generación $Y$, con la que se describe a las personas nacidas entre 1981 y 1995. Mientras las generaciones baby boom y $X$ se caracterizarían por centrarse más en sí mismas y buscar la armonía y el éxito personal, los integrantes de la generación $Y$ serían la primera generación de internet y se habrían socializado en una cultura de la flexibilidad, la movilidad y la orientación a entornos plurales y polifacéticos (Twenge et al., 2010; Pilgaard, 2013). Estas últimas generaciones se desarrollaron en un contexto en que la Educación Física se encontraba ya plenamente integrada en la educación obligatoria, las instalaciones deportivas habían experimentado un extraordinario crecimiento y el deporte espectáculo había alcanzado sus máximas cuotas de desarrollo. De acuerdo con estos antecedentes esta investigación se plantea dos hipótesis referidas al efecto generacional. Por un lado, la hipótesis de que la práctica deportiva de cada generación se mantendrá estable en el tiempo $\left(H_{1}\right)$. Por otro lado, que en el contexto del aumento de la práctica deportiva registrado en las tres últimas décadas, las generaciones más jóvenes presentarán tasas más elevadas que las más mayores $\left(H_{2}\right)$.

En segundo lugar, la evolución de la práctica deportiva también puede ser explicada por la influencia del efecto de ciclo vital, según el cual las variaciones registradas pueden ser vinculadas a restricciones que se manifiestan en diferentes etapas de la vida cotidiana. La infancia, la adolescencia, la juventud, la adultez, la madurez y la vejez son etapas de la vida que se caracterizan por cambios relacionados con el cuerpo, así como por diversos grados de independencia/dependencia y preferencias/restricciones a la realización de actividades de ocio (Butcher, Lindner y Johns, 2002; Pilgaard, 2013).

La transición de la infancia a la adolescencia o primera juventud implica una menor dependencia de la familia y otras instituciones sociales que suele condicionar la continuidad de una práctica deportiva a la que se había accedido de manera obligatoria a través del sistema educativo (Butcher, Lindner y Johns, 2002). A partir de los dieciocho años se inicia una fase conocida como segunda juventud en la que se produce un tránsito al mundo del trabajo o a los estudios superiores, que suele afectar a las actividades deportivas. Los jóvenes se vuelven más independientes de su familia y círculo de amistades y el ocio se torna más individual. Aunque de los veinte a los treinta años pueden acometer grandes 
esfuerzos y su rendimiento físico es elevado, hay evidencia empírica del descenso de la práctica deportiva que se produce tras la adolescencia (Ispizua y Monteagudo, 2002: 277). En la edad adulta, el aumento de disponibilidad económica suele verse acompañado de una disminución de tiempo libre. En esta etapa suele producirse una inmersión en la vida laboral en paralelo a la formación de una familia propia, circunstancias ambas que amplían las obligaciones y comprimen el tiempo libre. La llegada de los hijos supone una reorientación de las actividades que ahora pasan a focalizarse en ellos. En la medida que los hijos crecen y se vuelven menos dependientes se recobra una cierta independencia vital, hasta el punto de que es más probable disponer de tiempo para actividades deportivas en esta etapa que cuando se está construyendo una carrera profesional o criando hijos (Hirvensalo, Lampinen y Rantanen, 1998). Sin embargo, a partir de los cuarenta y cinco años se produce un cierto declive biológico que limita la práctica deportiva a actividades físicamente menos exigentes. Finalmente, tras la jubilación las personas disponen de más tiempo para la actividad deportiva, aunque a partir de los sesenta años se acentúa la decadencia biológica y, por tanto, las limitaciones físicas son mayores. De acuerdo con todo ello se plantea una tercera hipótesis - la existencia de un efecto de ciclo vital - según la cual se espera que, a medida que se dejen atrás las diversas etapas del ciclo vital, se produzca un descenso de la práctica deportiva $\left(H_{3}\right)$.

En tercer lugar, la práctica deportiva de los individuos puede también ponerse en relación con tendencias generales o acontecimientos específicos que se registran en una sociedad en un momento determinado, los llamados efectos de periodo. Se produce un efecto de periodo cuando los cambios se producen al mismo tiempo en todas las generaciones o etapas del ciclo vital (Pilgaard, 2013: 37). Una crisis económica, la universalización del sistema educativo o el auge del discurso sobre los beneficios del deporte para la salud son circunstancias que pueden producir un efecto de periodo en la práctica deportiva (Hirvensalo, Lampinen y Rantanen, 1998; Scheerder, Vanreusel y Taks, 2005; Breuer y Wicker, 2009).

Un hito central en la reciente historia española se encuentra en la Constitución española de 1978 y en la aprobación dos años después de la Ley General de la Cultura Física y el Deporte (Ley 13/1980). Esta ley reconocía el derecho de todo ciudadano a la educación física y su puesta en vigor supuso la progresiva dotación de instalaciones deportivas en los centros de educación obligatoria, así como el apoyo a la construcción de infraestructuras deportivas de uso público. La década de los noventa se abrió con la promulgación de una nueva ley - Ley 10/1990 del Deporte - que derogaba la anterior para centrarse en la regulación profesional del deporte de alta competición. Se consolidaba de ese modo el nuevo énfasis que las políticas deportivas iban a conferir al deporte de alto rendimiento, tal y como ya se había puesto de manifiesto tres años antes con la creación del Programa de la Asociación de Deportes Olímpicos (ADO). Solo dos años después se celebraron los Juegos Olímpicos de Barcelona (1992), que marcaron un punto de inflexión en el deporte español no solo por los éxitos cosechados sino también porque trajeron consigo un aumento de recursos para el deporte olímpico y de competición. Se iniciaba así un camino que fructificaría mediada la primera década del siglo XXI en lo que algunos han considerado como la edad dorada del deporte español (Llopis-Goig, 2016: 313), caracterizada por un amplio número de triunfos y victorias en numerosas modalidades deportivas. De acuerdo con esta información se plantea la cuarta y última hipótesis referida al efecto periodo, según la cual con el paso del tiempo se producirá un aumento de la práctica deportiva en todas las generaciones $\left(\mathrm{H}_{4}\right)$. 
TABLA 1. Encuestas incluidas en el diseño de la investigación

\begin{tabular}{cccc}
\hline Año & Muestra & Universo & Referencia \\
\hline 1984 & 2.474 & 18 y más años & E-1411 \\
1989 & 2.668 & 15 y más años & E-1814 \\
1993 & 2.500 & 65 y más años & E-2072 \\
1994 & 2.596 & 15 a 24 años & E-2105 \\
1994 & 2.491 & 25 y más años & E-2107 \\
1995 & 4.271 & $15-65$ años & E-2198 \\
1997 & 2.493 & 18 y más años & E-2266 \\
2000 & 5.160 & $15-75$ años & E-2397 \\
2005 & 8.170 & $15-75$ años & E-2599 \\
2010 & 8.925 & $15 y$ más años & E-2833 \\
2014 & 2.485 & 18 y más años & E-3029 \\
\hline
\end{tabular}

Fuente: Elaboración propia.

\section{Diseño de LA INVESTIGACIÓN}

\section{Fuentes}

De las 11 encuestas a las que se recurrió para esta investigación, 4 forman parte de la serie de 7 realizadas por el Centro de Investigaciones Sociológicas (CIS) entre 1980 y 2010 por encargo del Consejo Superior de Deportes (CSD), concretamente, las encuestas de 1995, 2000, 2005 y 2010 (véase la tabla 1)2. Además, se recurrió a otras 7 encuestas que incluían información sobre práctica deportiva que han permitido cubrir los años 1984, 1989, 1993, 1994, 1997 y 2014. En conjunto, las 11 encuestas contienen un periodo de treinta años (1984-2014), por lo que se cumple con creces la recomendación de Breuer y Wicker (2009) de abarcar un periodo temporal mínimo de veinte años.

\footnotetext{
2 Los microdatos de las encuestas de hábitos deportivos realizadas por el CIS por encargo del CSD en 1980, 1985 y 1990 no se encuentran disponibles. Tampoco se conservan los microdatos de las encuestas realizadas en 1968 y 1975 por el Instituto Nacional de Estadística (INE) y Gallup España, respectivamente, para la Delegación Nacional de Deportes.
}

Las encuestas mencionadas han sido analizadas por el profesor Manuel García Ferrando desde una perspectiva de análisis transversal o de tendencias en numerosas publicaciones. Sin embargo, no se ha efectuado nunca un análisis de cohortes, pese a que la serie de datos disponible lo permite tanto por las características técnicas de las encuestas realizadas (tamaño de la muestra, muestreo, operacionalización de la variable y procedimiento de contacto) como por el periodo temporal acumulado. Los rangos de edad disponible permiten un análisis de treinta años para las personas nacidas entre 1910 y 1969, de los últimos veinticinco años para las nacidas entre 1970 y 1979, y de los últimos catorce años para las nacidas en la década de los ochenta.

\section{Muestra}

Una vez configurada la matriz de datos, el número total de casos ascendió a 42.830. Como muestra la tabla 2, los tamaños muestrales superan el millar de casos en todos los grupos generacionales, con la excepción de las personas nacidas antes de 1910. En el 


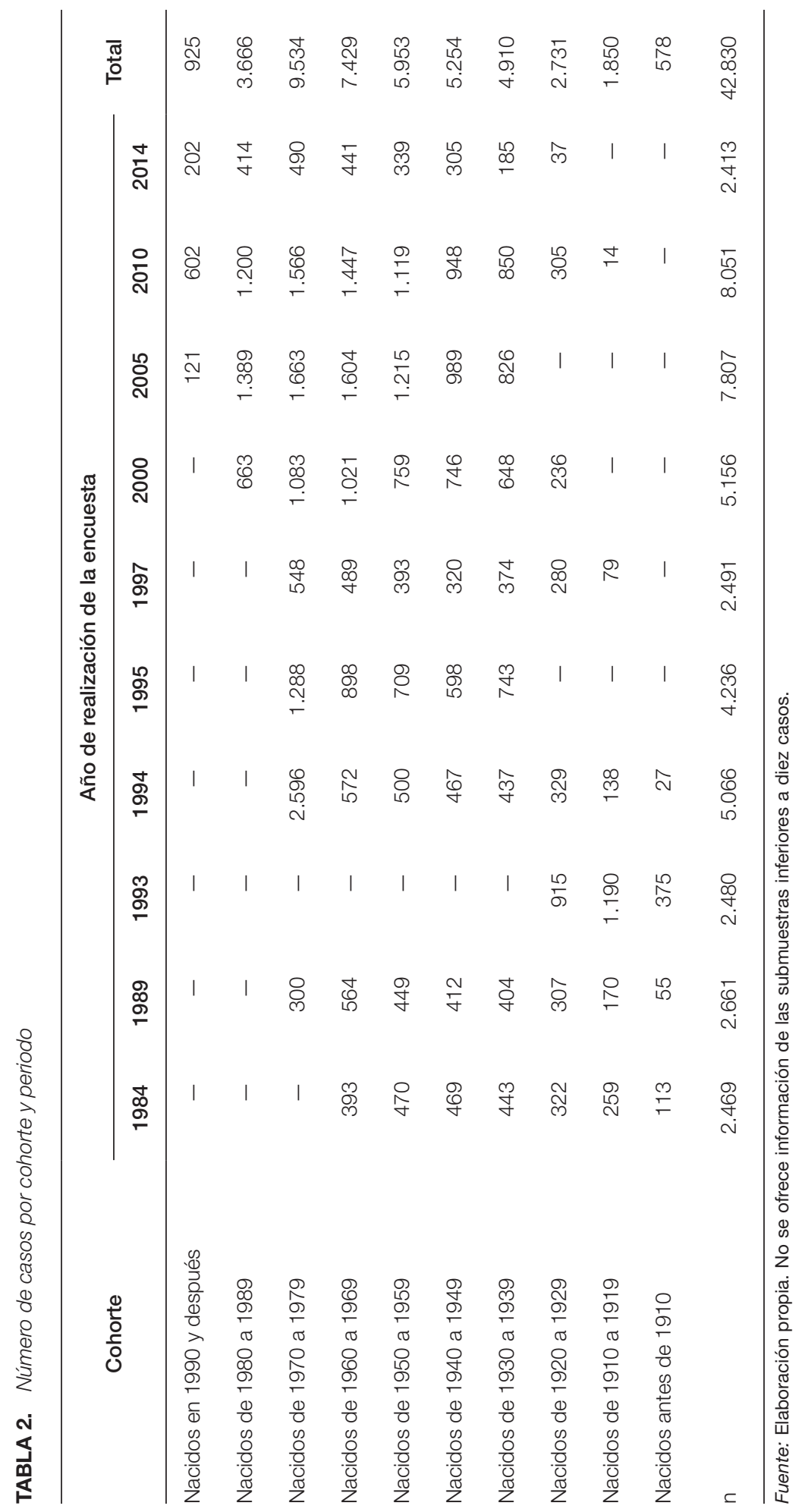

Reis. Rev.Esp.Investig.Sociol. ISSN-L: 0210-5233. № 157, Enero - Marzo 2017, pp. 85-102 
caso de los nacidos a partir de 1990 la cifra es cercana al millar $(n=925)$.

\section{Variables}

La variable dependiente de esta investigación ha sido la práctica deportiva. El modo en que esta variable se encontraba operacionalizada en las once encuestas utilizadas difería, por lo que se realizaron diversas transformaciones para mantenerla como variable dicotómica $(1=$ practica deporte, $0=$ no practica deporte) en todas las encuestas. Por lo que se refiere a las variables independientes, la variable adoptada para el ciclo vital ha sido la edad (15 y más años), mientras que para el periodo se ha recurrido al año de realización de la encuesta. La variable generación ha sido construida a partir de la combinación de las dos anteriores (edad * periodo), representando el efecto multiplicativo de la edad y el periodo, tal y como se ha hecho en otras investigaciones previas (Breuer y Wicker, 2009; Pilgaard, 2013). El efecto generacional es un efecto residual que se obtiene descontando el efecto periodo y el efecto ciclo vital (Jaime, 2008: 76). Así, la probabilidad de que una persona participe de manera regular en alguna forma de actividad deportiva es una función de la influencia del momento en que se ha registrado el dato (efecto de periodo), el efecto relativo a la edad que tiene en ese momento (efecto de ciclo vital) y el efecto de formar parte de un determinado grupo de edad en un periodo temporal concreto (efecto periodo).

\section{Técnicas estadísticas}

Las asociaciones entre la práctica deportiva y el resto de variables (edad, generación y periodo) han sido abordadas por medio de los estadísticos Chi-square y Gamma (Pilgaard, 2013: 40). La prueba Chi-square de Pearson es adecuada para medir la asociación entre dos variables nominales. El coeficiente Gamma $(\gamma)$ de Goodman y Kruskall es una medida del grado y el tipo de asociación entre dos variables cualitativas en escala ordinal que toma valores en el intervalo $[-1,1]$. Mientras las variables temporales de esta investigación pueden ser consideradas cuanto menos variables de tipo ordinal, la variable práctica deportiva fue codificada como dummy.

Por lo que se refiere al análisis bivariable se ha adoptado la misma estrategia que en otros estudios previos. La $H_{1}$ ha sido examinada a partir de un análisis horizontal de las cohortes, lo que ha permitido identificar la evolución de las pautas de cada generación a lo largo del tiempo. La $\mathrm{H}_{2}$ ha sido estudiada a través de un análisis en diagonal que ha hecho posible comparar las diferencias entre los grupos de la misma edad pertenecientes a diferentes cohortes generacionales. En el caso de la $\mathrm{H}_{3}$, a través de un análisis vertical que ha permitido analizar los niveles de participación deportiva en los diferentes grupos de edad en diez momentos entre 1984 y 2014 . Por último, la $H_{4}$ ha implicado el estudio de los cambios a través del análisis de la evolución global así como de la evolución en cohortes específicas a nivel horizontal (Pilgaard, 2013: 40-41).

Los análisis bivariables se han complementado con un análisis de regresión logística binaria que ha examinado las hipótesis $\mathrm{H}_{2}, \mathrm{H}_{3}$ y $\mathrm{H}_{4}$ de manera conjunta. La $\mathrm{H}_{2}$ ha sido analizada tomando las generaciones y los periodos como variables continuas, la $\mathrm{H}_{3}$ tomando la edad en años, y en el caso de la $\mathrm{H}_{4}$, en periodos. Para evitar posibles riesgos de multicolinealidad las variables independientes fueron recodificadas y centradas 3 (Pilgaard, 2013: 41; Aiken y West, 1991).

\footnotetext{
3 La variable edad fue centrada asignando el valor $-43,88$ a la edad mínima y $+43,88$ a la máxima (dado que la edad media de la muestra total era de 43,88 años). En cuanto a la variable periodo, el centrado se realizó asignando el valor -15 al primer año de la serie $y+15$ al último (ya que el periodo alcanzaba un total de treinta años). La elaboración de la variable generación, por último, se calculó mediante el producto de la edad centrada y el periodo centrado.
} 


\section{Resultados}

\section{Análisis de los efectos generacionales}

La tabla 3 muestra los porcentajes de práctica deportiva de las diez cohortes generacionales estudiadas desde 1984 a 2014. El análisis horizontal de la tabla permite rechazar la $H_{1}$. Los contrastes de Chi-square son significativos para siete de las diez cohortes generacionales estudiadas, lo que significa que estas no muestran una conexión estable con la práctica deportiva a lo largo del periodo 1984-2014. La excepción son los nacidos entre 1980 y 1989 , entre 1920 y 1929 y antes de 1910 que, por el contrario, exhiben una mayor estabilidad en el tiempo. Habría que tener en cuenta, no obstante, que la primera y la tercera de esas tres cohortes solo cuentan con cuatro momentos temporales, lo que puede estar condicionando los resultados. Así pues, la $H_{1}$ quedaría rechazada al haberse detectado variaciones significativas en la práctica deportiva de la mayoría de las generaciones estudiadas.

El contraste de la $\mathrm{H}_{2}$ requirió un análisis en diagonal de las tasas de práctica deportiva4. Los datos permiten afirmar que las generaciones más jóvenes tienen unas tasas de práctica deportiva más elevadas que las más mayores. En el periodo 1984-2014 se produce un aumento de la práctica deportiva en las generaciones de edades medias. En 1984 , las personas que en ese momento tienen entre 25 y 64 años presentan tasas del $34,22,3,11,5$ y $6,8 \%$. En 2014 , las cuatro cohortes que se encuentran en ese mismo tramo de edad alcanzan tasas del 58, 49,4, 32,9 y $33,3 \%$, lo que significa un aumento medio de 25 puntos. También es relevante el incremento que se observa en las dos generaciones más mayores al principio y al final

\footnotetext{
4 La expresión en diagonal no debe tomarse al pie de la letra dado que el rango de edad cubierto por las cohortes no se corresponde con las distancias en años entre los diez momentos temporales incluidos en la investigación.
}

del periodo: en 1984 las personas que tienen de 65 a 74 años y más de 74 presentan tasas de práctica deportiva del 4,6 y 2,7\% respectivamente. Las dos generaciones que tienen esa misma edad en 2014 obtienen unas tasas del 21 y 13,5\% respectivamente, por lo que en treinta años se produce un incremento de 14 puntos. En los años siguientes, sin embargo, esa tendencia pierde gran parte de su intensidad. Las personas que en 1984 tenían entre 15 y 24 años presentaban una tasa del $52,9 \%$, mientras que las personas que tenían esa misma edad dos décadas después alcanzaban una tasa del 56,2\%. Por otro lado, las personas que tenían menos de veinte años en 1989 presentaban una tasa del 57,3\%, mientras que veinte años después, las personas con esa misma edad alcanzan un $60,5 \%$. Ambos hechos podrían estar indicando la existencia de un punto de inflexión en la evolución de la práctica deportiva en virtud de la cual las generaciones más jóvenes habrían pasado a ser menos activas en términos físico-deportivos. Esta tendencia encaja con lo apuntado en el marco teórico cuando se señalaba que las últimas generaciones se habían socializado de un modo mucho más flexible y desarrollado vinculaciones menos estables, en un contexto en el que la oferta de actividades de ocio es mucho más amplia. En resumen, la hipótesis $\mathrm{H}_{2}$ quedaría verificada para las personas nacidas antes de los años ochenta pero puesta en entredicho para las nacidas en esa década y con posterioridad.

\section{Análisis de los efectos de ciclo vital}

La caída de la práctica deportiva tras el final de la adolescencia ha sido apuntada por numerosas investigaciones. Este hecho queda claramente confirmado por los datos de la tabla 3, que ponen de manifiesto que la práctica deportiva desciende significativamente desde las etapas juveniles a las de mayor edad de manera sistemática en los diez momentos temporales incluidos en la investiga- 
ción. La $\mathrm{H}_{3}$, por tanto, queda confirmada.

Sin embargo, si se repara en los valores del coeficiente gamma a lo largo de la serie, se puede comprobar que su peso se reduce progresivamente. Mientras el valor más alto del periodo se registra en $1984(\gamma=-0,613)$, en los últimos años se obtienen los más bajos ( $\gamma=-0,394$ en 2005). De modo que aunque la asociación estadística entre el ciclo vital y la práctica deportiva es significativa, la evolución del coeficiente gamma podría considerarse como un indicio del progresivo debilitamiento de la relación entre ambas variables. Esto es lo que explica que mientras la cohorte nacida entre 1960 y 1969 presenta una tasa del 39,2\% en el año 2010 -que supera en más de 7 unidades porcentuales a la obtenida por la generación de nacidos entre 1950 y 1959 ese mismo año (31,7\%)-, cuatro años después las posiciones se inviertan al alcanzar estos últimos una tasa superior a la de los primeros $(33,3 \%$ frente a $32,9 \%)$.

Estos resultados podrían estar relacionados con la influencia combinada de los efectos generacionales, de ciclo vital y de periodo. Si se observa la tabla 3 se puede constatar que la generación nacida entre 1950 y 1959 prácticamente tiene la misma tasa de práctica deportiva al inicio que al final del periodo (34\% en 1984 y 33,3\% en 2014). La generación posterior (nacidos entre 1960 y 1969), sin embargo, experimenta una fuerte caída: empieza la serie con 52,9\% y acaba con $32,9 \%$. Esto podría deberse a la existencia de diferencias entre ambas generaciones, según las cuales la preocupación por el cuerpo y la salud, por ejemplo, se habría activado de manera más prematura en la generación nacida entre 1950 y 1959. El aumento de la tasa de práctica deportiva de esa generación entre 2010 y 2014 -desafiando la habitual tendencia a la disminución- podría estar relacionado con el momento en que los adultos - tras la emancipación de sus hijos - quedan libres de las tareas y obligaciones propias de la pa- ternidad y disponen de más tiempo propio (Hirvensalo, Lampinen y Rantanen, 1998; Ispizua y Monteagudo, 2002; Breuer y Wicker, 2009; Pilgaard, 2013).

En definitiva, los datos examinados confirman la relación lineal negativa entre edad y práctica deportiva: a medida que aquella aumenta y se superan las diversas etapas del ciclo vital disminuye de manera significativa la práctica deportiva. Ahora bien, se observa un progresivo debilitamiento de la relación entre ambas variables así como una menor discontinuidad de la práctica deportiva entre distintas fases del ciclo vital en los últimos años de la serie. Ambos extremos podrían estar relacionados - tal como se ha apuntado en el marco teórico - con un mayor grado de sensibilización en torno a la salud y el cuidado del cuerpo que ha proliferado en una determinada fase y que habría tenido una mayor influencia en personas de mayor edad, lo que supondría una combinación del efecto periodo con el efecto generacional.

\section{Análisis de los efectos de periodo}

La lectura horizontal de la tabla 3 revela que las generaciones nacidas antes de 1960 han incrementado su tasa de práctica deportiva entre 1984 y 2014. Así, pese a que su edad ha ido aumentando con el paso del tiempo también lo ha hecho su actividad deportiva. Esta tendencia - que con anterioridad ha sido considerada como una evidencia empírica de la inexistencia de unas pautas generacionales estables en el tiempo y ha conducido al rechazo de la $H_{1}-$, podría ser considerada como un síntoma de la existencia de efectos de periodo. Una prueba de ello la proporciona el análisis de los resultados del coeficiente gamma que - al margen de que en algunos casos concretos no es significativo - registra un signo positivo para todas las cohortes nacidas antes de 1960. Así, por ejemplo, las personas nacidas entre 1920 y 1929 registran en 1984 una tasa del 
$6,8 \%$, mientras que en el último año que aparecen en la serie alcanzan un 9,8\%. Lo mismo sucede con la generación nacida entre 1930 y 1939: en 1984 tiene una tasa del $11,5 \%$ y en 2014 alcanza un 13,5\% (tras haber llegado a superar el 16\% con anterioridad). La generación nacida entre 1940 y 1949 registra en 1984 una tasa del 22,3\%, solo ligeramente superior a la de 2014 (21\%), si bien en 2010 alcanza un 28,3\%. Por último, en el caso de la generación nacida entre 1950 y 1959, el crecimiento de la práctica deportiva es ya mucho más débil, hasta el punto de que los porcentajes al inicio y al final de la serie son muy similares (34\% en 1984 y 33,3\% en 2014). La evolución señalada, sin embargo, no se registra entre las generaciones más jóvenes. Las personas nacidas entre 1960 y 1969 registran una tasa del 52,9\% en 1984 que cae hasta el 32,9\% en 2014. En cuanto a las personas nacidas entre 1970 y 1979 , pasan del 57,3\% de 1989 al $49,4 \%$ de 2014. En la medida en que estos resultados desmienten parcialmente la tradicional relación lineal negativa entre edad y práctica deportiva, podrían estar indicando la existencia de un efecto de periodo que habría afectado especialmente a las personas de mayor edad. Dado que estas cohortes son las primeras que han gozado de un mayor nivel de estudios y se encuentran más sensibilizadas ante la necesidad de mantenerse físicamente activas en fases avanzadas, han incrementado sus tasas de actividad a medida que aumentaba su edad. El efecto periodo, en este caso, consistiría en un aumento de la práctica que estaría relacionado con la mayor preocupación por la salud y el desarrollo de unos estilos de vida activos que se habría ido diseminando en determinados grupos de la sociedad en las últimas décadas hasta convertirse en una suerte de norma social.

Más allá de este análisis general, el examen horizontal de la tabla 3 permite identificar varios efectos de periodo que conviene mencionar. El primero se produjo en el tramo que abarca desde la aprobación de la Constitución en 1978 hasta la llegada al gobierno del Partido Socialista Obrero Español en 1982. Esta etapa incluiría aspectos tan relevantes como la aprobación de la Ley General de Cultura Física y Deporte de 1980 y la construcción de un amplio número de instalaciones deportivas. Aunque los microdatos de las encuestas de esos años no se encuentran disponibles, existen algunas referencias a los mismos que pueden resultar esclarecedoras. La tasa de práctica deportiva de las personas nacidas en los años treinta pasó del $10 \%$ en 1968 al $17 \%$ en 1974 , mientras que la de las nacidas en los cuarenta pasó del 18\% en 1974 al 22,3\% en 1984 (Martínez del Castillo et al., 1992). Estos datos ponen de manifiesto que, al menos en esas dos cohortes, en el periodo de transición a la democracia se produjo un aumento de la práctica deportiva que contrarrestó el descenso de actividad que suele conllevar el aumento de edad.

Un segundo efecto de periodo se habría producido desde 1984 hasta 1995, cuando la mayor parte de las instalaciones deportivas ya se había construido y la sociedad española vivía el entusiasmo de la celebración de los Juegos Olímpicos de Barcelona (1992). Durante ese periodo, las tasas de actividad deportiva aumentaron en prácticamente todas las cohortes, por lo que puede hablarse de un segundo efecto de periodo. Las personas nacidas antes de 1920 pasaron del 2,7\% de 1984 al 3,7\% de 1994, mientras que las nacidas en la década de los diez y los veinte pasaron del 4,6 y el $6,8 \%$ al 8,7 y el $10,3 \%$ respectivamente en ese mismo periodo. Por otro lado, las tres generaciones posteriores (nacidos en los treinta, cuarenta y cincuenta) pasaron del $11,5,22,3$ y $34 \%$ de 1984 al 14,5, 26,3 y 36,1\% de 1995. Por último, las personas nacidas en los sesenta y setenta pasaron del 37 y el $57,3 \%$ de 1989 al 46 y $59,7 \%$ de 1995 . Se trata, pues, de un segundo efecto de periodo en virtud del cual todas las generaciones aumentaron su prác- 


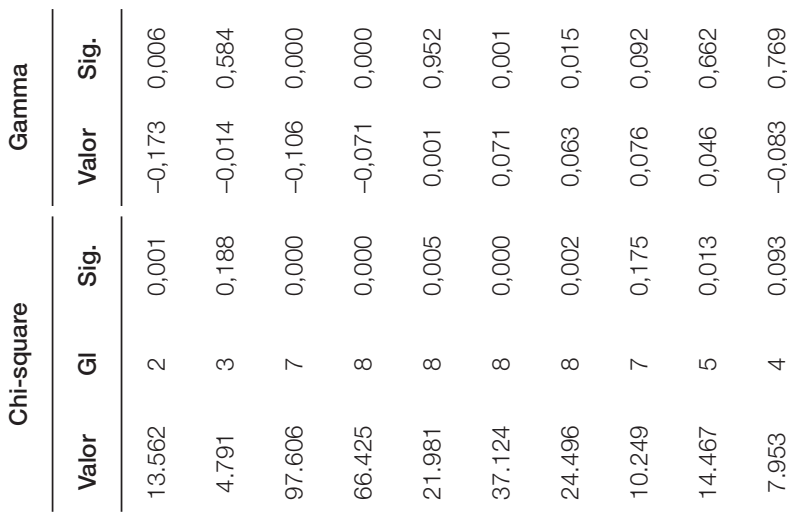

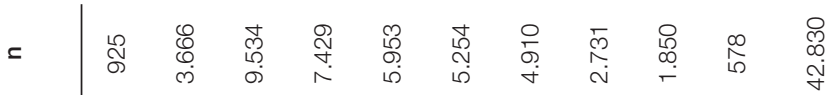

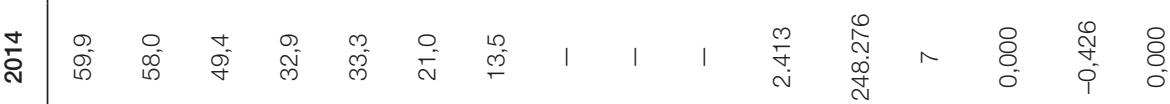

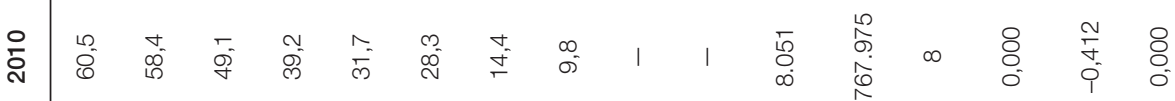

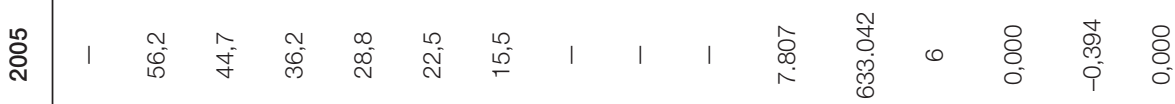

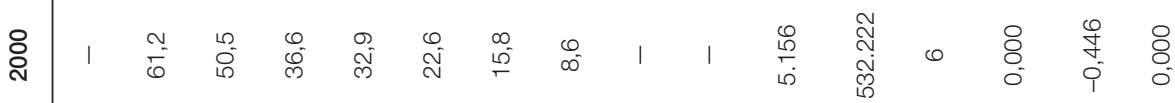

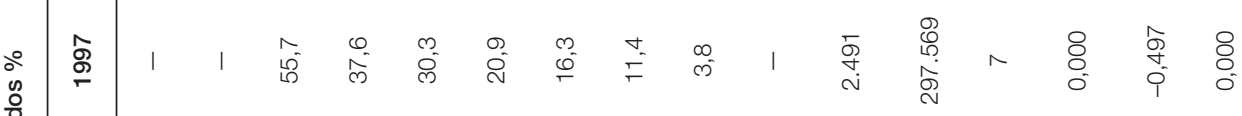

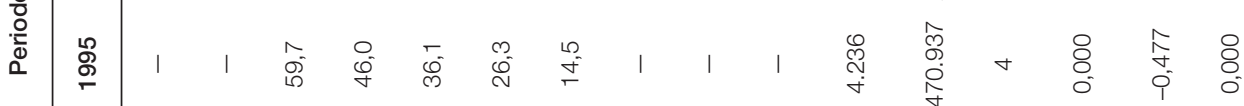

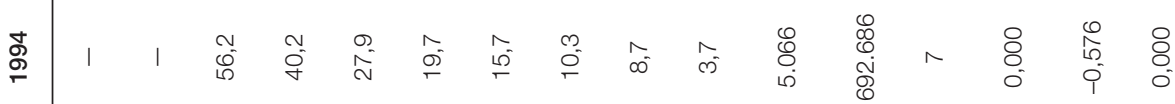

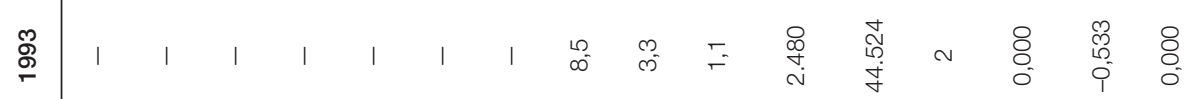

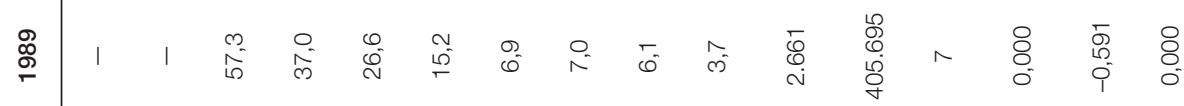

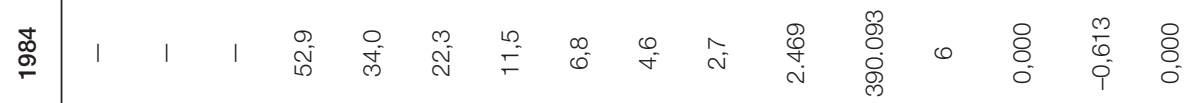

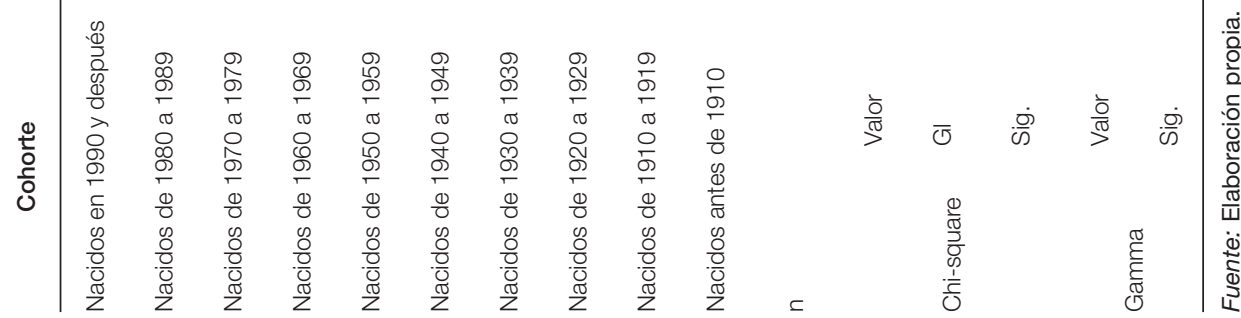


tica deportiva en contra de la comúnmente admitida tendencia a reducirla con el aumento de la edad.

Un tercer efecto de periodo se habría manifestado en la primera década del siglo XXI y estaría relacionado con la progresiva difusión de un estilo de vida activo entre las personas de mayor edad, en virtud del cual las personas nacidas en los años treinta, cuarenta y cincuenta habrían mantenido estable su tasa de actividad física-deportiva una vez cumplidos los sesenta, los cincuenta y los cuarenta, respectivamente. En el primer caso registraban un $15,8 \%$ en el 2000 y un $14,4 \%$ en el 2010; en el segundo, un 22,6 y un $28,3 \%$ respectivamente; y en el tercero, un 32,9 y un 33,3\%, también respectivamente. Las tres generaciones, pues, mantienen su actividad en fases medias y avanzadas de su ciclo vital, lo que pone de manifiesto la existencia de un efecto periodo relacionado con el auge y mayor difusión de la cultura de la salud, el cuidado del cuerpo y el ejercicio físico que ha tenido mayor influencia en estas cohortes, probablemente porque se encuentran en una fase de su vida en que se han liberado de diversas responsabilidades y disponen de más tiempo libre.

\section{Análisis multivariable}

Se ha realizado por último un análisis de regresión logística binomial con el objeto de poner nuevamente a prueba las hipótesis de esta investigación mediante la elaboración de un modelo que examina de manera conjunta la influencia de los tres efectos. Como ya se ha señalado anteriormente, la variable dependiente fue dicotomizada ( 1 = practica deporte, $0=$ no practica deporte). Respecto a las variables independientes, en primer lugar, se consideró que las únicas variables que debían incluirse en el modelo eran las referidas a los tres efectos cuya influencia se pretendía medir 5 . En segundo lugar, no ha sido necesario transformar ninguna de ellas en una variable dummy dado que originariamente eran métricas (ciclo vital) u ordinales (periodo y generación). En cualquier caso, las tres fueron sometidas a un proceso de centrado para evitar posibles riesgos de multicolinealidad. En tercer lugar, las correlaciones bivariables entre las variables independientes no mostraron riesgos de multicolinealidad, pues los coeficientes de correlación de Pearson fueron siempre inferiores al 0,60 y por tanto se encontraban lejos del umbral del 0,80 (Cea D’Ancona, 2002: 132).

En la tabla 4 se muestran los resultados de la ecuación de regresión logística. Por lo que se refiere a la bondad de ajuste, el valor obtenido para la $\mathrm{R}^{2}$ de Nagelkerke es de 0,197 . Por otro lado, en el $69,4 \%$ de los casos el valor observado en la variable dependiente coincide con el valor pronosticado por el modelo.

Si se presta atención a los exponentes de cada coeficiente $\mathrm{B}^{6}$, la primera observación que cabe extraer a partir de los resultados del modelo es que los tres efectos (periodo, ciclo vital y generación) tienen una influencia altamente significativa sobre la variable dependiente. El efecto periodo es el más potente, seguido del efecto ciclo vital y, por último, del efecto generación. Esto indica que los efectos de periodo han sido los más importantes en la evolución de la práctica deportiva de los españoles entre 1984 y 2014.

\footnotetext{
5 Se seguía de ese modo la sugerencia de Cea D'Ancona (2002: 131) cuando insiste en la necesidad de que el modelo analítico se encuentre correctamente especificado.

6 Esta es la opción más aconsejable habida cuenta de que su interpretación se asemeja a la de los coeficientes de regresión lineal. El cambio en la variable dependiente asociado a un cambio de una unidad en la independiente, manteniéndose el resto de las variables independientes constante, expresa el cambio en odds (razón de probabilidad de ocurrencia/no ocurrencia de un evento) cuando la variable independiente aumenta en una unidad (Cea D'Ancona, 2002: 157).
} 
TABLA 4. Regresión logística

\begin{tabular}{lccccccccc}
\hline & B & S.E. & Wald & df & Sig. & Exp(B) & 95\% C.I. for $\operatorname{Exp(B)}$ \\
\cline { 7 - 10 } & & & & & & & & Lower & Upper \\
\hline Periodo & 0,056 & 0,002 & 728,449 & 1 & 0,000 & 1,057 & 1,053 & 1,061 \\
Ciclo vital & $-0,046$ & 0,001 & 4729,021 & 1 & 0,000 & 0,955 & 0,953 & 0,956 \\
Generación & 0,001 & 0,000 & 143,188 & 1 & 0,000 & 1,001 & 1,001 & 1,001 \\
Constante & $-1,507$ & 0,019 & 6601,033 & 1 & 0,000 & 0,221 & & \\
\hline
\end{tabular}

Función de verosimilitud $(-2$ Log likelihood) $=48.406,071$.

R2 de Nagelkerke $=0,197$. Eficacia predictiva $=69,4 \%$.

Los efectos de ciclo vital muestran un signo negativo mientras que los efectos de periodo son de carácter positivo y muestran más potencia que los de ciclo vital. Los efectos de periodo positivos causan un aumento o evitan un descenso de la participación deportiva de diversas generaciones en una medida que compensa o supera el efecto del ciclo vital, un hallazgo que también ha sido identificado en Dinamarca (Pilgaard, 2013) y Alemania (Breuer y Wicker, 2009).

Los resultados de la regresión logística confirman las tres hipótesis para las que se realizó, esto es, la $\mathrm{H}_{2}$, la $\mathrm{H}_{3}$ y la $\mathrm{H}_{4}$ y suponen, por tanto, una confirmación añadida a la obtenida previamente a través de los análisis bivariables. Estos resultados ponen de manifiesto la influencia de los tres efectos en la evolución de la práctica deportiva. Debe señalarse asimismo que, aunque las $\mathrm{R}^{2}$ y los coeficientes $B$ arrojaban puntuaciones no muy elevadas, esto es habitual en los estudios de participación deportiva, por lo que resulta más relevante detenerse en el análisis del nivel de significatividad estadística de los modelos (Scheerder y Vos, 2011). Debe tenerse en cuenta que las tres variables incluidas en el modelo no son en realidad buenos predictores de la varianza total de la participación/no participación deportiva (Pilgaard, 2013: 45).

\section{Conclusiones}

Este estudio ha puesto de manifiesto que la práctica deportiva de las distintas cohortes generaciones estudiadas en la investigación no se ha mantenido estable a lo largo del periodo, sino que se ha visto sometida a variaciones significativas. Desde esta perspectiva se ha rechazado la $H_{1}$, que postulaba la estabilidad de las pautas de participación deportiva de las distintas generaciones en virtud de la influencia permanente que las actitudes y comportamientos cristalizados en el periodo de socialización primaria supondrían en su desarrollo posterior (efecto generacional). Este hecho se ha interpretado como una prueba de que los efectos de periodo y ciclo vital son más potentes que los de carácter generacional, algo que, por otro lado, no puede sorprender habida cuenta de los grandes cambios experimentados por el deporte en la sociedad española durante las tres décadas analizadas - consideración del deporte como derecho ciudadano, desarrollo de políticas públicas deportivas y aumento del número de instalaciones deportivas (Llopis-Goig, 2016).

En segundo lugar, los análisis realizados han permitido corroborar la $\mathrm{H}_{2}$ - una hipótesis también vinculada al efecto generacional - al haberse podido constatar que con el 
paso del tiempo la práctica deportiva que presentan las personas de una determinada edad aumenta con respecto a la tasa que registraban las personas de esa misma edad en un momento anterior. El análisis multivariable ha corroborado esta hipótesis aunque ha cuantificado su influencia en un grado inferior al identificado para los efectos periodo y ciclo vital. El análisis bivariable ha confirmado dicha hipótesis para las personas nacidas antes de los ochenta, pero no para las nacidas en esa década y después, al ser ese el momento en que se produce un punto de inflexión en la evolución de la práctica deportiva en virtud del cual las personas más jóvenes dejan de presentar incrementos de la misma con respecto a las personas de la misma edad de décadas anteriores. Se trata de una tendencia ya detectada en otras investigaciones europeas (Sheerder y Vos, 2011; Pilgaard, 2013) que encontraría explicación en el hecho de que las cohortes más jóvenes se habrían socializado en contextos de mayor pluralismo, flexibilidad y orientación al cambio y habrían desarrollado unas pautas menos estables de vinculación con la actividad deportiva (Twenge et al., 2010). La confirmación de esa tendencia en el futuro plantearía por primera vez un cambio en la evolución de la práctica deportiva en la medida en que podría implicar una reducción de la actividad deportiva por parte de las generaciones más jóvenes y, por tanto, una cierta separación de los beneficios derivados de un estilo de vida activo. Sin embargo, también habría que tener en cuenta que las tasas de partida de estas generaciones más jóvenes son superiores a las de las generaciones de más edad $y$, por tanto, el interrogante que queda es si la socialización deportiva que han vivido en edades tempranas ha dejado suficiente huella como para influir en futuras etapas de sus vidas una vez superado el habitual desenganche del deporte organizado en la etapa adolescente (Coakley, 2015).

En tercer lugar, la investigación ha verificado la $H_{3}$ (efecto de ciclo vital), según la cual a medida que aumenta le edad disminuye la práctica deportiva, ya que en la medida que se van atravesando las distintas fases del ciclo vital se producen cambios relacionados con el cuerpo, así como modificaciones en las relaciones de independencia/dependencia que pueden afectar a las preferencias o a las restricciones de las personas en relación a sus actividades de ocio. No obstante, se ha detectado un debilitamiento creciente de la relación entre la edad y la práctica deportiva que podría estar relacionado con una mayor sensibilidad de las personas de edades medias y avanzadas hacia el cuidado del cuerpo, así como un mayor compromiso con unos estilos de vida saludables y físicamente activos (Hirvensalo, Lampinen y Rantanen, 1998; Breuer y Wicker, 2009). Esa mayor sensibilidad y compromiso con un estilo de vida activo sería fruto de un efecto periodo que estaría teniendo una mayor influencia en las generaciones de mayor edad a las que estaría llevando a mantener sus tasas de actividad físico-deportiva una vez superados los cincuenta y los sesenta años.

En cuarto lugar, los análisis realizados han confirmado también la $\mathrm{H}_{4}$. Por un lado, el análisis de regresión logística ha evidenciado que los efectos de periodo han sido los de mayor influencia en la evolución de la práctica deportiva de los españoles entre 1984 y $2014\left(H_{4}\right)$, seguidos de los de ciclo vital $\left(H_{3}\right)$ y los de generación $\left(H_{2}\right)$. Los efectos generacionales han sido, pues, confirmados en línea con lo previsto por la $H_{2}$, pero su impacto ha resultado ser menor al de los de ciclo vital $\left(H_{3}\right)$, que a su vez se ven superados por los de periodo $\left(H_{4}\right)$. Y ello es así porque estos últimos (los efectos de periodo) han impactado de manera constante en todas las cohortes generacionales y en distintos momentos del ciclo vital, poniendo de manifiesto que el impacto combinado de las tendencias socioculturales (estilos de vida, cultura del cuerpo y salud) y las políticas públicas (instalaciones deportivas, programas 
de promoción deportiva) en la población puede suponer un desafío a las restricciones que aparecen con la edad y modificar los intereses y preferencias que supuestamente habían cristalizado en los procesos de socialización primaria. Es especialmente relevante a este respecto el aumento de planes, actividades e instalaciones orientadas de manera específica a la actividad física de las personas mayores que se han llevado a cabo y desarrollado en gran parte de los municipios españoles.

Por otro lado, y aún en relación con la $\mathrm{H}_{4}$, los análisis bivariables efectuados han proporcionado una mejor comprensión del modo en que han actuado los efectos de periodo al identificar la existencia de tres intervalos específicos. El primero se habría producido entre los años 1975 y 1984 y estaría relacionado con la aprobación de la Constitución en 1978 y la puesta en vigor de la Ley 13/1980 General de la Cultura Física y el Deporte, que supuso la consideración del deporte como un derecho ciudadano y el aumento de las instalaciones deportivas. El segundo se habría producido entre 1984 y 1995 y se explicaría por el amplio apoyo e inversión dedicado al deporte ante la celebración de los Juegos Olímpicos de Barcelona en 1992, así como por el éxito deportivo que los mismos supusieron para los deportistas españoles. El tercero se habría producido ya en la primera década del siglo XXI y habría tenido como protagonistas a las personas de edad media y avanzada que estarían manteniendo sus tasas de actividad física una vez alcanzados los cuarenta, los cincuenta y los sesenta años por la influencia de una nueva cultura de la salud, el bienestar y la calidad de vida.

\section{Bibliografía}

Aiken, Leona S. y West, Stephen G. (1991). Multiple Regressions: Testing and Interpretation Interactions. Thousand Oaks, California: SAGE Publications.
Breedveld, Koen y Hoekman, Remco (2011). «Measuring Sports Participation in the Netherlands. The Need to Go Beyond Guidelines». European Journal for Sport and Society, 8(1/2): 117-132.

Breuer, Christoph (2005). «Cohort Effects in Physical Inactivity. A Neglected Category and Its Health Economical Implications». Journal of Public Health, 13(4): 189-195.

Breuer, Christoph y Wicker, Pamela (2009). «Decreasing Sports Activity with Increasing Age? Findings from a 20-year Longitudinal and Cohort Sequence Analysis». Research Quarterly for Exercise and Sport, 80(1): 22-31.

Butcher, Janice; Lindner, Koenraad J. y Johns, David P. (2002). «Withdrawal from Competitive Youth Sport: A Retrospective Ten-year Study». Journal of Sport Behaviour, 25: 145-163.

Cea D’Ancona, María Á. (2002). Análisis multivariable. Teoría y práctica en la investigación social. Madrid: Síntesis.

Coakley, Jay (2015). Sports in Society. Issues and Controversies. New York: McGraw-Hill.

Engel, Claudia y Nagel, Siegfried (2011). «Sports Participation During the Life Course». European Journal for Sport and Society, 8(1/2): 45-63.

García-Ferrando, Manuel y Llopis-Goig, Ramón (2011). Ideal democrático y bienestar personal. Encuesta de Hábitos Deportivos en España 2010. Madrid: Centro de Investigaciones Sociológicas.

Gratton, Chris; Rowe, Nick y Veal, A. J. (2011). «International Comparisons of Sports Participation in European Countries: An Update of the COMPASS Project». European Journal for Sport and Society, 8(1/2): 99-116.

Hirvensalo, Mirja; Lampinen, Piiivi y Rantanen, Taina (1998). "Physical Exercise in Old Age. An Eightyear Follow-up Study on Involvement, Motives and Obstacles Among Persons Age 65-84». Journal of Aging and Physical Activity, 6: 157-168.

Ispizua, Marian y Monteagudo, María J. (2002). «Ocio y deporte: nuevas claves para el desarrollo humano». En: García Ferrando, M.; Lagardera, F. y Puig, N. (comps.). Sociología del deporte. Madrid: Alianza.

Jaime, Antonio M. (2008). «Trayectorias de participación política de la juventud europea: ¿Efectos de cohorte o efectos de ciclo vital?». Revista de Estudios de Juventud, 81: 67-93. 
Klostermann, Claudia y Nagel, Siegfried (2014). "Changes in German Sport Participation: Historical Trends in Individual Sports». International Review for the Sociology of Sport, 49(5): 609634.

Llopis-Goig, Ramón (2016). «El sistema deportivo español. Estructura organizativa y pautas de participación ciudadana». En: Llopis-Goig, R. (ed.). La participación deportiva en Europa. Políticas, culturas y prácticas. Barcelona: UOC.

Martínez del Castillo, Jesús; Navarro, Carlos; Fraile, Antonio; Puig, Nuria; Jiménez, Pedro; Martínez, Javier y de Miguel, Carlos (1992). Deporte, sociedad y empleo. Madrid: Ministerio de Educación y Ciencia.

McPherson, Barry D.; Curtis, James E. y Loy, John W. (1993). The Social Significance of Sport. Champaign, Illinois: Human Kinetics.

Pilgaard, Maja (2013). «Age Specific Differences in Sports Participation in Denmark. Is Development Caused by Generation, Life Phase or Time Period Effects?». European Journal for Sport and Society, 10(1): 31-52.

Scheerder, Jeroen; Vanreusel, Bart y Taks, Marijke (2005). «Stratification Patterns of Active Sport
Involvement Among Adults. Social Change and Persistence». International Review for the Sociology of Sport, 40(2): 139-162.

Scheerder, Jeroen y Vos, Steven (2011). «Social Stratification in Adult's Sports Participation from a Time-trend Perspective. Results from a 40-year Household Study». European Journal for Sport and Society, 8(1/2): 31-44.

Scheerder, Jeroen; Vandermeerschen, Hanne; Van Tuyckom, Charlotte; Hoekman, Remco; Breedveld, Koen y Vos, Steven (2011). Understanding the Game: Sport Participation in Europe: Facts, Reflections and Recommendations. Disponible en: http://hdl.handle.net/1854/LU-1932490, acceso 27 de julio de 2015.

Twenge, Jean M.; Campbell, Stacy M.; Hoffman, Brian J. y Lance, Charles E. (2010). «Generational Differences in Work Values: Leisure and Extrinsic Values Increasing, Social and Intrinsic Values Decreasing". Journal of Management, 36(5): 1117-1142.

Yang, Yang (2011). «Aging, Cohorts and Methods». En: Binstock, R. H. y George, L. K. (eds.). Handbook of Aging and the Social Sciences. Amsterdam: Elsevier.

RECEPCIÓN: 28/07/2015

REVISIÓN: $14 / 01 / 2016$

APROBACIÓN: 31/05/2016 



\section{Life Phase, Generation and Period Effects on Sports Participation in Spain (1984-2014)}

Efectos de ciclo vital, generación y periodo en la práctica deportiva de los españoles (1984-2014)

Ramón Llopis-Goig

Key words

Age

- Life Phase Effect

- Generational Effect

- Period Effect

- Sports Practice

- Time

\section{Palabras clave}

Edad

- Efecto de ciclo vital

- Efecto generación

- Efecto periodo

- Práctica deportiva

- Tiempo

\begin{abstract}
This article presents an analysis of the influence of generational, life phase and period effects on the evolution of sports participation in Spain. The analysis refers to the period between 1984 and 2014 and it was conducted using an APC (age, period and cohort) design application. This study verifies the impact of the life phase effect on sports practice and the existence of strong periodical effects at three specific moments, as well as the greater intensity of these two effects as compared to the generational effects. The analysis reveals that period effects have had the greatest influence on the evolution of sports participation between 1984 and 2014.
\end{abstract}

\section{Resumen}

Esta investigación presenta un análisis de la influencia de los efectos generacionales, de ciclo vital y de periodo en la evolución de la práctica deportiva de la población española. El análisis se ha referido al periodo 1984-2014 y se ha llevado a cabo mediante la aplicación de un diseño APC (age, period and cohort). El estudio ha constatado el impacto del efecto de ciclo vital sobre la práctica deportiva y la existencia de fuertes efectos de periodo en tres tramos concretos, así como la mayor intensidad de estos dos efectos frente a los de carácter generacional. Los análisis efectuados también han evidenciado que los efectos de periodo han sido los más influyentes en la evolución de la práctica deportiva entre 1984 y 2014.

\section{Citation}

Llopis-Goig, Ramón (2017). "Life Phase, Generation and Period Effects on Sports Participation in Spain (1984-2014)”. Revista Española de Investigaciones Sociológicas, 157: 85-102. (http://dx.doi.org/10.5477/cis/reis.157.85)

Ramón Llopis-Goig: Universidad de Valencia | ramon.llopis@uv.es 


\section{INTRODUCTION $^{1}$}

Over recent decades, sports participation has undergone a notable increase in most European countries (Scheerder et al., 2011). This has also been the case in Spain, where studies have revealed that the percentage of individuals engaging in sporting activities has increased from 12\% in 1968 (Martínez del Castillo et al., 1992) to $47 \%$ in 2014 (Llopis-Goig, 2016). A fundamental aspect in this increase is related to the incorporation of elderly individuals in this sporting practice. In Spain, for example, although the relationship between age and sporting practice continues to be linear and negative (García-Ferrando and Llopis-Goig, 2011), a change has been perceived. In 2014, groups between the ages of 55 and 64 and 65 and older registered rates of 33 and $17 \%$ respectively, whereas three decades earlier, the first group reached only $4 \%$ and the second group was not involved at all in this practice (Llopis-Goig, 2016). In other European countries however, the trend has been much more intense and an increase in age no longer implies a decrease in sporting activity (Breedveld and Hoekman, 2011; Gratton, Rowe and Veal, 2011).

These conclusions refer mainly to cross sectional studies that have been conducted with the main limitation of failing to specify whether or not the relative differences in age were caused by generation or life cycle effects. In order to identify the changes produced over time as individuals pass through distinct life phases, a longitudinal panel design is necessary, in which the same sample is interviewed successive times over an extensive time period. The data resulting from studies based on longitudinal research designs permit the analysis of intra-individual

\footnotetext{
1 The study described in this article was conducted thanks to research funding (modality A) awarded by the 2014 Research Support Subsidies of the Spanish Social Research Center (CIS).
}

changes and -in application to the study of sports participation - make it possible to obtain information on the specific history of dropouts and entries/re-entries of each individual in the sporting system. This data, however, is not available in Spain or in most European countries, although approximations may be made with a merging of diverse cross sectional surveys, known as a 'false panel'.

This study intends to offer an analysis of the development of sports participation over a time period of 30 years (1984-2014) based on an APC (age, period and cohort) or cohort analysis design (Yang, 2011: 17). The main objective was to identify the impact of generation effects, life cycle and period on the evolution of sporting practice in the Spanish population. It attempts to respond to the following three questions: How do age-related differences in sports participation patterns relate to the individual situations experienced across one's life?, Can the cohort patterns be explained by the influences of the social and historical circumstances that characterize the socialization and development processes of individuals?, and Are there any types of global influences that have determined the evolution of sporting practice in the Spanish population over the last thirty years?

The work contains an initial section in which the characteristics of the APC model are presented along with the theoretical approach and the study hypotheses. In the next section, the study design is presented as well as the technical characteristics of the research. Then, the most relevant results are revealed and finally, there is a conclusions section.

\section{Approach}

\section{The APC model}

Unlike the typical cross-sectional studies - which examine the relationship of a specific practice or behavior with individual fac- 
tors (such as gender, education level, labor situation or civil state) - , the studies that are encompassed under the APC denomination examine the origin and process of social change based on age (the life cycle effect), cohort (generation effect) and reference year (period effect), permitting the identification of how the levels of a specific behavior evolve in society.

The study of these effects in the field of sporting practice has been very limited on an international level and in regards to Spain, it is virtually inexistent. The retrospective studies of Engel and Nagel (2011) and Klostermann and Nagel (2014) regarding sporting practice across the life cycle have revealed the existence of a trend that is characterized by progressive drop out and entry/re-entry over the individual's life, which may be considered the result of changes that occur in their needs, demands and desires to participate upon entering new life cycle phases. Another major contribution was provided by Breuer and Wicker (2009), combining longitudinal analysis with cohort analysis in order to validate the age differences detected in prior cross-sectional studies. In their analysis - conducted in Germany, as was the case with Engel and Nagel (2011) - they found that cohort effects were the effects offering a major contribution to the development of sporting practice, and despite the passage of time, they remained constant and even increased -despite the effects of age increase - the level of sports practice within each cohort. Breuer and Wicker (2009) also referred to the effects of period, which would explain the increasing importance of health and would have modified the social norms referring to age, getting rid of the image of old age as a phase of physical inactivity. In this way, the effects of period would compensate and even surpass those of life cycle (age) as identified in the cross-sectional surveys. Another work conducted based on the APC analysis model is that of Maja Pilgaard (2013), which examined the evolution of sports participation in Denmark. The results obtained by Pilgaard highlight that sports participation of the Danish between 1975 and 2007 was affected by three types of effects, with period effects being the most important, followed by those of life cycle. The research presented in our study comes in response to studies such as those conducted by Engel and Nagel (2011), Klostermann and Nagel (2014), Breuer (2005), Breuer and Wicker (2009) and Pilgaard (2013), especially this latter, from which we adopted the hypothesis and analysis strategy, so as to apply it to Spanish society.

\section{Theoretical framework and hypotheses}

The generation effect hypothesis, also known as the cohort effect, is based on the idea that the sporting context in which each generation is socialized affects their subsequent beliefs and behavior patterns (McPherson, Curtis and Loy, 1993: 37; Coakley, 2015: 52). The generational cohorts considered in this study were socialized virtually throughout the entire 20th century and therefore they have enjoyed distinct possibilities of access to sporting practices.

The first generational group considered is the post-war generation, a group that includes those born in the 1920s and who were socialized in an under-developed society that lacked sporting facilities. The group known as the development generation includes individuals born in the 1930s and 1940s who lived through the effects of the Stabilization Plan of 1959 and the other two initial development plans. The radio allowed them to experience events such as the defeat of the English soccer team by Spain in the 1950 World Cup, the victory of Bahamontes in the Tour de France in 1959 or the 5 consecutive victories of the Real Madrid soccer team in the first five editions of the European Cup. Sporting practice, however, remained quite limited in a country that lacked basic sporting infrastructures. The label of the transition 
generation is typically attributed to those born in the 1950s, socialized in a period in which the country had begun to exit its period of isolation and was becoming integrated into the Western world. Sports were some of the main content of the mass media, even though sporting practice was not very popular. Those individuals born in the 1960s, known as the baby boomers, grew up in a period of transition from the Spanish dictatorship to democracy, in a period of cultural change that came with the boom of the television and increased cultural diversification. The so-called generation $X$ includes those born in the 1970s and who were socialized in a context that was marked by the fall of the Berlin Wall, European integration, advances in globalization and the boom of a new electronic and digital cultural paradigm. Then came generation $Y$, describing those born between 1981 and 1995. Although those from the baby boom and $X$ generations are characterized by focusing more on themselves and seeking out harmony and personal success, those of generation $Y$ would be the first generation of the Internet and they were socialized in a culture of flexibility, mobility and orientation towards plural and multi-faceted environments (Twenge et al., 2010; Pilgaard, 2013). These last generations developed in a context in which Physical Education was already fully integrated in mandatory education programs, sporting installations had experienced extraordinary growth and sporting events had reached their maximum development shares. Based on this background information, our study proposes two hypotheses referring to the generation effect. The first hypothesis is that sports practice for each generation remains stable over time $\left(H_{1}\right)$. Second, that in the context of the increasing sports practice recorded over the past three decades, the younger generations will have higher rates than the older ones $\left(H_{2}\right)$.

Second, the evolution of sports practice may also explained by the influence of the life cycle effect, according to which the recorded variations maybe related to restrictions that appear over the different stages of everyday life. Childhood, adolescence, young adulthood, adulthood, maturity and old age are life phases that are characterized by changes related to the body, as well as by diverse degrees of independence/dependence and preferences/restrictions to the carrying out of leisure activities (Butcher, Lindner and Johns, 2002; Pilgaard, 2013).

The transition from childhood to adolescence or early youth implies a decreased dependence on the family and other social institutions that tend to condition the continuity of a sporting practice which may have been carried out as a mandatory part of the education system (Butcher, Lindner and Johns, 2002). Upon turning eighteen, a phase known as the second youth takes place in which there is the transfer to the world of employment or higher studies, which tends to affect the participation in sporting activities. Young people now become more independent from their family and circle of friends and leisure activities become more individualized. Although between the twenties and thirties, great efforts may be made and physical performance is high, empirical evidence suggests a decrease in sporting practice that occurs after adolescence (Ispizua and Monteagudo, 2002: 277). In the adult age, an increased economic availability tends to be accompanied by a decrease in free time. During this phase, there tends to be an immersion in the working life that parallels the creation of one's own family, with both circumstances increasing obligations and reducing free time. The arrival of children means a re-directing of one's activities that now tend to focus on them. As their children grow up and become less dependent, they recover certain independence, so that they are likely to have more time for sporting activities in this phase than when creating a professional career or raising children (Hirvensalo, Lampinen and Rantanen, 1998). However, once turning forty-five, a cer- 
tain biological decline takes place which limits sporting practice to less demanding physical activities. Finally, after reaching the retirement age, individuals have more time for sporting activities, although as of the sixties, a biological decline is evident and therefore, physical limitations are greater. According to all of this, a third hypothesis has been proposed -the existence of a life cycle effectaccording to which it is anticipated that, as the diverse life phases go by, a decrease takes place in sporting activity $\left(H_{3}\right)$.

Third, sporting practice in individuals may also be related to general trends or specific events that occur in a society at a specific time, the so-called period effects. A period effect takes place at the same time for all generations or phases of the life cycle (Pilgaard, 2013: 37). An economic crisis, the universalization of the education system or a boom in discourse the benefits of sports for health are all circumstances that may produce a period effect in sporting practice (Hirvensalo, Lampinen and Rantanen, 1998; Scheerder, Vanreusel and Taks, 2005; Breuer and Wicker, 2009).

A major milestone in recent Spanish history took place with the creation of the Spanish Constitution of 1978 and the approval, two years later, of the Law on Physical Culture and Sports (Act 13/1980). This law recognized the right of all citizens to physical education and its implementation led to the progressive creation of mandatory sporting facilities in schools, as well as the support of the construction of public sporting infrastructures. The 1990s began with the enacting of a new law -Act 10/1990 on Sports - which repealed the previous law, so as to focus on professional regulation of competitive sports. In this way, the new emphasis that sports policies were going to grant to high performance sports was consolidated, as declared three years previously with the creation of the Program of the Olympic Sports Association (ADO). Only two years later, the Olympic Games were held in Barcelona (1992), a turning point in Spanish sports, not only due to the successes achieved but also due to the increased resources for Olympic sports and competitions. Thus, a path was created which would flourish over the first decade of the 21 st century, which some considered to be the golden age of Spanish sports (Llopis-Goig, 2016: 313), characterized by a large number of wins and victories in numerous sports. In accordance with this information, a fourth and final hypothesis was proposed, referring to the period effect, according to which, with the passage of time, there shall be an increase in the sporting practice in all generations $\left(H_{4}\right)$.

\section{Research design}

\section{Sources}

Of the 11 surveys that were used for the study, 4 form a part of the series of 7 conducted by the Sociological Research Center (CIS, based on its initials in Spanish) between 1980 and 2010, as requested by the Spanish Sports Council (CSD), specifically, the surveys from 1995, 2000, 2005 and 2010 (see Table 1)2. Furthermore, 7 other surveys were used which included information on sporting practices covering the years 1984, 1989, 1993, 1994, 1997 and 2014. Altogether, the 11 surveys cover a period of 30 years (19842014), complying with the recommendation of Breuer and Wicker (2009) of including a minimal time period of twenty years.

The mentioned surveys have been analyzed in numerous publications by Professor Manuel García Ferrando using a cross-sectional or trend analysis perspective. However, a cohort analysis has been yet to be conducted, despite the fact that the available series of data would permit this analysis, given the

\footnotetext{
2 Microdata from the surveys on sporting habits conducted by the CIS upon request of the CSD in 1980, 1985 and 1990 are not available. Also unavailable were the microdata from surveys conducted in 1968 and 1975 by the National Statistics Institute (INE) and Gallup España, respectively, for the National Sports Delegation.
} 
TABLE 1. Surveys included in the research design

\begin{tabular}{cccc}
\hline Year & Sample & Universe & Reference \\
\hline 1984 & 2,474 & 18 and older & E-1411 \\
1989 & 2,668 & 15 and older & E-1814 \\
1993 & 2,500 & 65 and older & E-2072 \\
1994 & 2,596 & 15 to 24 years & E-2105 \\
1994 & 2,491 & 25 and older & E-2107 \\
1995 & 4,271 & $15-65$ years & E-2198 \\
1997 & 2,493 & 18 and older & E-2266 \\
2000 & 5,160 & $15-75$ years & E-2397 \\
2005 & 8,170 & $15-75$ years & E-2599 \\
2010 & 8,925 & 15 and older & E-2833 \\
2014 & 2,485 & 18 and older & E-3029 \\
\hline
\end{tabular}

Source: author's own creation.

technical nature of the surveys conducted (sample size, sampling, operationalization of the variable and contact procedure), as well as the accumulated time period. The age ranges available permit an analysis of thirty years for individuals born between 1910 and 1969 , of the last twenty-five years for those born between 1970 and 1979, and of the last fourteen years for those born in the 1980s.

\section{Sample}

Once configuring the data matrix, the total number of cases reached 42,830. As shown in Table 2, sample sizes exceeded one thousand cases in all generation groups, except in those born before 1910. In the case of those born after 1990, the figure is close to one thousand $(n=925)$.

\section{Variables}

The dependent variable of this study is the sports practice. The way in which this variable was operationalized in the eleven surveys differs, therefore various transformations were carried out in order to maintain it as a dichoto- mous variable $(1=$ sporting practice, $0=$ no sporting practice) in all of the surveys. As for the independent variables, the variable adopted for the life cycle was age (15 years and older), while for period the year of survey completion was used. The generation variable was constructed based on the combination of the two previous (age * period), representing the multiplicative effect of age and period, as done in other prior studies (Breuer and Wicker, 2009; Pilgaard, 2013). The generation effect is a residual effect that is obtained by deducting the period effect and the life cycle effect (Jaime, 2008: 76). Thus, the probability that an individual participates regularly in some type of sporting activity is a function of the influence of the time when said data was recorded (period effect), the relative effect of their age at this time (life cycle effect) and the effect of forming a part of a specific age group in a specific time period (period effect).

\section{Statistical techniques}

The associations between the sporting practice and the other variables (age, generation and period) have been examined with Chi- 


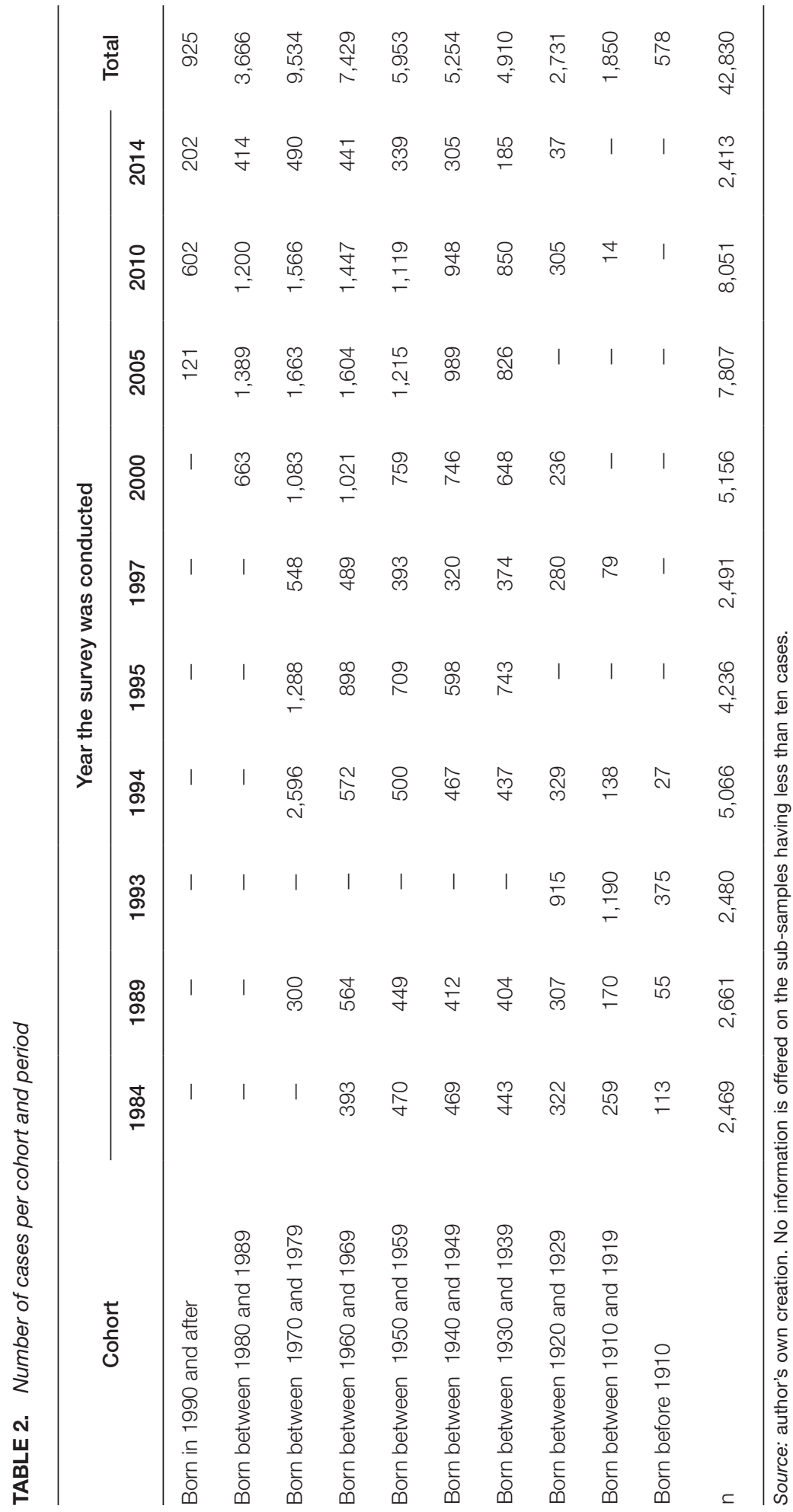

Reis. Rev.Esp.Investig.Sociol. ISSN-L: 0210-5233. Nº 157, January - March 2017, pp. 85-102 
square and Gamma statistics (Pilgaard, 2013: 40). Pearson's Chi-square test is appropriate for measuring the association between two nominal variables. The Gamma $(\gamma)$ coefficient of Goodman and Kruskall is a measure of degree and type of association between two qualitative variables in an ordinal scale, taking values in the interval $[-1,1]$. While the time variables of this study may be considered to be ordinal, to say the least, the sports practice variable was coded as dummy.

As for bivariate analysis, the same strategy was adopted as in other prior studies. $H_{1}$ was examined based on a horizontal analysis of the cohorts, permitting identification of the evolution of the patterns of each generation over time. $\mathrm{H}_{2}$ was studied through a diagonal analysis that makes it possible to compare the differences between the groups of the same age belonging to different generational cohorts. In the case of $H_{3}$, a vertical analysis permitted analysis of the levels of sporting participation in the different age groups ten times between 1984 and 2014. Finally, $H_{4}$ involved the study of changes through the analysis of the overall change, as well as the change in specific cohorts at a horizontal level (Pilgaard, 2013: 40-41).

The bivariate analysis was complemented with a binary logistics regression that collectively examined the $\mathrm{H}_{2}, \mathrm{H}_{3}$ and $\mathrm{H}_{4}$ hypotheses. $\mathrm{H}_{2}$ was analyzed by using the generations and periods as continuous variables; $H_{3}$ was examined by using the age in years and for $H_{4}$ periods were used. In order to prevent potential risks of multicollinearity, the independent variables were recoded and centered 3 (Pilgaard, 2013: 41; Aiken and West, 1991).

\footnotetext{
3 The age variable was centered, assigning the value -43.88 to the minimum age and +43.88 to the maximum age (given that the mean age of the overall sample was 43.88 years). As for the period variable, the centering was carried out by assigning the value -15 to the first year of the series and +15 to the last (given that the period attained a total of 30 years). The creation of the generation variable, finally, was calculated using the product of centered age and centered period.
}

\section{Results}

\section{Analysis of generation effects}

Table 3 reveals the percentages of sporting practice for the ten generational cohorts studied between 1984 and 2014. The horizontal analysis of the table allows for the rejection of $H_{1}$. The Chi-square contrasts are significant for seven of the ten generational cohorts studied, meaning that they do not reveal a stable connection with the sporting practice across the 1984-2014 period. The exception is those born between 1980 and 1989, between 1920 and 1929 and before 1910 which, on the contrary, revealed a greater stability over time. It should be considered, however, that the first and third of these three cohorts have only four time moments that may condition the results. Thus, $H_{1}$ is rejected given that significant variations were detected in the sporting practice for the majority of the generations that were studied.

The contrast of $\mathrm{H}_{2}$ required a diagonal analysis of the rates of sports practice 4 . The data permitted us to affirm that the youngest generations had higher rates of sports practice than the older generations. In the 19842014 period, there was an increase in sports practice in the generations of average ages. In 1984, the individuals who, at this time were between 25 and 64 years of age presented rates of $34,22.3,11.5$ and $6.8 \%$. In 2014 , the four cohorts found in this same age range attained rates of 58, 49.4, 32.9 and $33.3 \%$, meaning a mean increase of 25 points. The increase observed in the two oldest generations at the beginning and end of the period is also relevant: in 1984, individuals between 65 and 74 years of age and those over 74 had sports practice rates of 4.6 and $2.7 \%$ respectively. The two generations having

\footnotetext{
4 The expression in diagonal should not be taken literally, given that the age range covered by the cohorts does not correspond to the distances in years between the ten time moments included in the study.
} 
these same ages in 2014 obtained rates of 21 and $13.5 \%$ respectively, meaning that in 30 years, there was an increase of 14 points. In the following years however, this trend lost much of its intensity. Individuals who in 1984 were between 15 and 24 years of age had a rate of $52.9 \%$ while those of this same age two decades later reached a rate of $56.2 \%$. On the other hand, individuals under the age of twenty in 1989 presented a rate of $57.3 \%$ whereas twenty years later, individuals with this same age attained a rate of $60.5 \%$. Both of these facts may indicate the existence of a turning point in the evolution of sporting practice by virtue of which the youngest generations went on to become less active in terms of physical-sports activity. This trend fits in with that suggested in the theoretical framework when it was suggested that the most recent generations socialize in a more flexible manner and develop less stable links, in a context in which the leisure activity offer is much broader. To summarize, the $\mathrm{H}_{2}$ hypothesis is verified for those individuals born prior to the 1980s, but is challenged for those born in this decade and later.

\section{Analysis of life cycle effects}

The decline in sporting practice after the end of adolescence has been pointed out by numerous studies. This fact is clearly confirmed by the data shown in Table 3, which highlights that sports practice decreases significantly after the youth phases to those of adulthood, in a systematic manner in the ten time moments included in the study. $\mathrm{H}_{3}$, therefore, is confirmed.

However, if the gamma coefficient values are repaired across the series, it may be confirmed that its weight is progressively reduced. Meanwhile, the highest value for the period was recorded in $1984(\gamma=-0.613)$ obtaining the lowest values over recent years $(\gamma$ $=-0.394$ in 2005). So, although the statistical association between life cycle and sports practice is significant, the evolution of the gamma coefficient may be considered to be an index of the progressive weakening of the relationship between these two variables. This explains why, although the cohort born between 1960 and 1969 has a rate of $39.2 \%$ in 2010 -exceeding the rate obtained for the generation born between 1950 and 1959 for the same year $(31.7 \%)$ by over 7 percentage units - only four years later, the positions inverted, with the latter reaching a rate that exceeded that of the former $(33.3 \%$ as compared to $32.9 \%$ ).

These results may be related to the combined influence of the generation, life cycle and period effects. Table 3 suggests that the generation born between 1950 and 1959 has practically the same rate of sports practice at the start as at the end of the period (34\% in 1984 and $33.3 \%$ in 2014). The subsequent generation (born between 1960 and 1969), however, experienced a strong decline: it began the series at $52.9 \%$ and ended it at $32.9 \%$. This may be due to the existence of differences between both generations in terms of concerns over body and health. For example, it would have been activated in a more premature manner in the generation born between 1950 and 1959. The increase in sporting practice rate for this generation between 2010 and 2014 -challenging the usual trend to decline - may be related with the time at which the adults - after their children's emancipation - are freed of the tasks and obligations of parenthood and therefore have more time for themselves (Hirvensalo, Lampinen and Rantanen, 1998; Ispizua and Monteagudo, 2002; Breuer and Wicker, 2009; Pilgaard, 2013).

Ultimately, the data that was examined confirms the negative linear relationship between age and sports practice: as it increases and the diverse life cycle stages pass, sports practice declines significantly. Meanwhile, a progressive weakening is observed in the relationship between both variables as well as a lower discontinuity of the sporting practice between the distinct life cycle phas- 
es in the last years of the series. Both extremes may be related - as suggested in the theoretical framework - with an increased degree of awareness of health and care for the body which took place during a specific phase and would have had a greater influence on older individuals, suggesting a combination of period and generation effects.

\section{Analysis of period effects}

The horizontal reading of Table 3 reveals that the generations born before 1960 increased their rate of sports practice between 1984 and 2014. Thus, despite the fact that their age increased over time, their sports activity also increased. This trend - which was previously considered to be empirical evidence of the lack of stable generational patterns over time and has led to the rejection of $\mathrm{H}_{1}-$, may be considered to be a symptom of the existence of period effects. This is tested by the analysis of the gamma coefficient results which - regardless of the fact that in some specific cases is not significant - had a positive sign for all of the cohorts born prior to 1960. Thus, for example, those born between 1920 and 1929 had a rate of $6.8 \%$ in 1984 whereas in the last year appearing in the series, they reached $9.8 \%$. The same occurred with the generation born between 1930 and 1939: in 1984, it had a rate of $11.5 \%$ and in 2014, it reached $13.5 \%$ (after previously exceeding 16\%). In 1984, the generation born between 1940 and 1949 reached a rate of $22.3 \%$, only slightly superior to that of 2014 (21\%), while in 2010 , it reached $28.3 \%$. Finally, in the case of the generation born between 1950 and 1959, the growth of sports practice is already much weaker until reaching the point where the scores at the start and end of the series are quite similar (34\% in 1984 and $33.3 \%$ in 2014). The suggested evolution however, is not found for the youngest generations. Individuals born between 1960 and 1969 had a rate of $52.9 \%$ in 1984 which decreased to $32.9 \%$ in 2014 . As for those born between 1970 and 1979, it went from $57.3 \%$ in 1989 to $49.4 \%$ of 2014 . As these results partially refute the traditional negative linear relationship between age and sports practice, it may be suggesting the existence of a period effect that would have especially affected the older individuals. Given that these cohorts are the first to have a higher education level and are more aware of the need to remain physically active at more advanced phases, their activity rates have increased as their age increases. The period effect, in this case, consists of an increase in the practice that would be related to an increased concern over health and the development of some active lifestyles that would have extended over certain groups of society during the last decades until becoming a sort of social norm.

Beyond this general analysis, the horizontal analysis of Table 3 allows for the identification of various period effects that should be mentioned. The first took place in the period between the Constitution's approval in 1978 until the governing of the Spanish Socialist Party in 1982. This phase includes such relevant aspects as the approval of the Law on Physical Culture and Sports of 1980 and the construction of a large number of sporting facilities. Although the microdata from the surveys for these years are not available, there are some references to the same that may prove enlightening. The rate of sports practice in individuals born in the 1930 went from $10 \%$ in 1968 to $17 \%$ in 1974 , while that for those born in the 1940s went from 18\% in 1974 to 22.3\% in 1984 (Martínez del Castillo et al., 1992). This data reveals that at least for these two cohorts, in the period of transition to the democracy, an increase took place in in sporting practice which offset the decrease in activity that tends to take place with the ageing process.

A second period effect would have taken place between 1984 and 1995, when the majority of the sporting facilities had already 


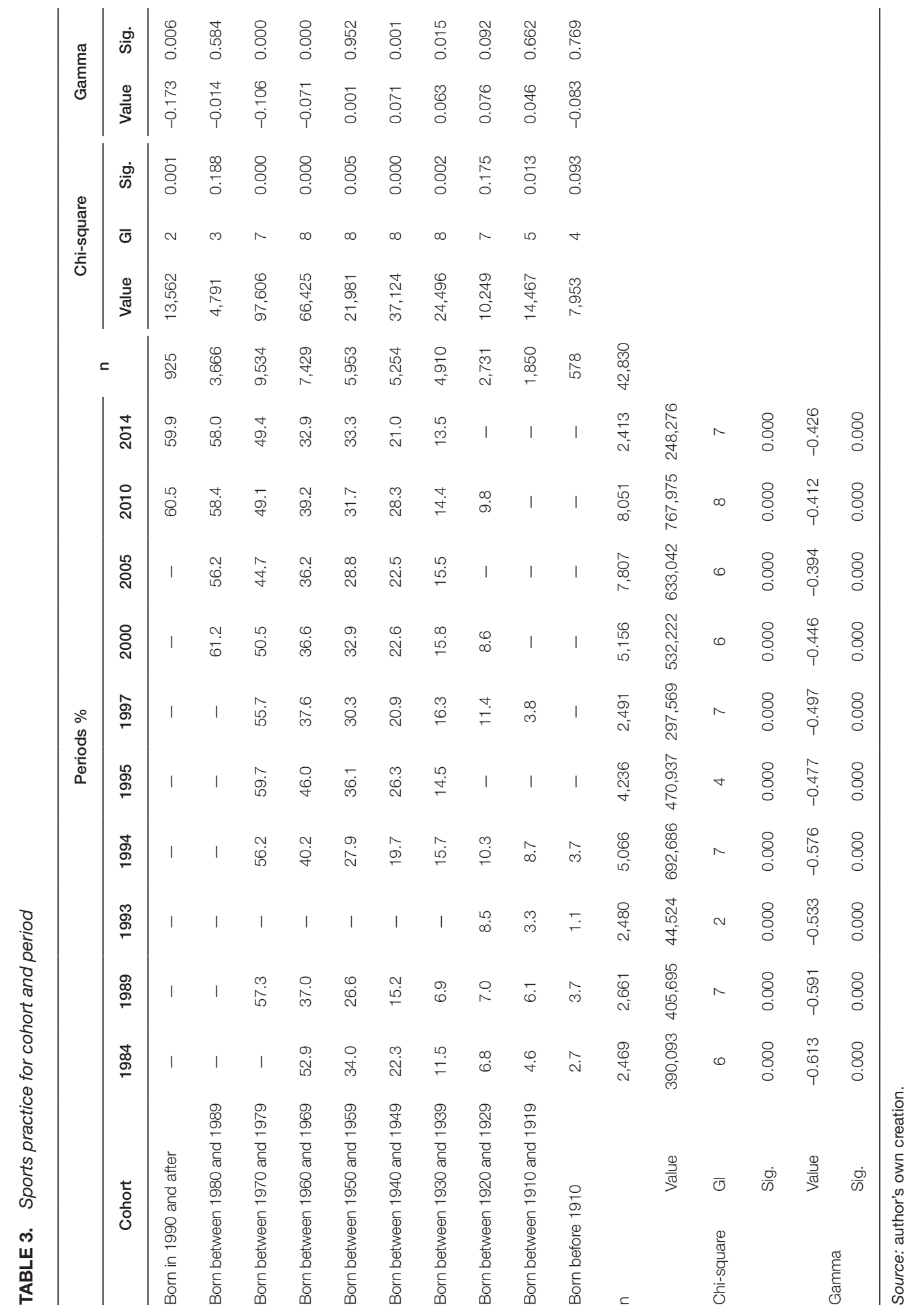


been constructed and Spanish society experienced the enthusiasm of the celebration of the Olympic Games in Barcelona (1992). During this period, the rates of sporting activity increased in almost all of the cohorts, thus it is possible to speak of a second period effect. Those individuals born prior to 1920 went from $2.7 \%$ in 1984 to $3.7 \%$ in 1994 while those born in the decade of 1910 and 1920 went from 4.6 and $6.8 \%$ to 8.7 and $10.3 \%$ respectively during this same period. On the other hand, the three subsequent generations (born in the thirties, forties and fifties) went from 11.5, 22.3 and 34\% in 1984 to $14.5,26.3$ and $36.1 \%$ in 1995 . Finally, those individuals born in the 1960s and 1970s had rates that increased from 37 and $57.3 \%$ in 1989 to 46 and $59.7 \%$ in 1995 . This is, therefore, a second period effect by virtue of which all of the generations increased their sporting practice despite the commonly admitted tendency to reduce the same with age.

A third period effect may have appeared in the first decade of the $21^{\text {st }}$ century and would be related to the progressive diffusion of an active lifestyle in the older individuals, thus those born in the 1930s, 1940s and 1950s maintained their rate of physicalsporting activity once reaching sixty, fifty and forty years of age, respectively. In the first care, they registered $15.8 \%$ in 2000 and $14.4 \%$ in 2010; in the second, they had a rate of 22.6 and $28.3 \%$ respectively; and the third group reached 32.9 and $33.3 \%$ respectively. The three generations therefore, maintained their physical activity in the middle and advanced phases of their life cycle, highlighting the existence of a period effect that is related to the boom and increased dissemination of the culture of health, care for the body and exercise, which has had an increased influence on these cohorts, probably because they were in phases of their life in which they were free of diverse responsibilities and had more free time.

\section{Multivariate analysis}

Finally, a binomial logistic regression analysis was conducted in order to once again challenge the research hypothesis through the creation of a model that collectively examines the influence of the three effects. As previously mentioned, the dependent variable was dichotomized ( $1=$ sports practice, $0=$ no sports practice). As for the independent variables, first, the only variables that should be included in the model were those referring to the three effects whose influence is to be measured5. Second, it has not been necessary to transform any of these into a dummy variable, given that originally they were metric (life cycle) or ordinal (period and generation). In any case, the three were subjected to a centering process in order to prevent the potential risk of multicollinearity. Third, the bivariate correlations between the independent variables do not reveal risks of multicollinearity, given that the Pearson correlation coefficients were always under 0.60 and therefore, were far from the threshold of 0.80 (Cea D'Ancona, 2002: 132).

Table 4 reveals the results of the logistic regression equation. In regards to the goodness of fit, the value obtained for Nagelkerke's $\mathrm{R}^{2}$ is 0.197 . On the other hand, in $69.4 \%$ of the cases, the value observed in the dependent variable coincides with the value that was predicted for the model.

If considering the exponents of each $\mathrm{B}^{6}$ coefficient, the first observation to be made based on the results of the model is that the

\footnotetext{
5 When following the suggestion offered by Cea D'Ancona (2002: 131) who insisted on the need for the analytical model to be correctly specified.

6 This is the most recommended option considering that its interpretation is similar to that of the linear regression coefficients. The change in dependent variable associated with a change of unit in the independent, keeping the other independent variables constant, expresses the change in odds (ratio of probability of occurrence/no occurrence of an event) when the independent variable increases in a unit (Cea D'Ancona, 2002: 157).
} 
TABLE 4. Logistic regression

\begin{tabular}{lccccccccc}
\hline & B & S.D. & Wald & df & Sig. & Exp(B) & \multicolumn{2}{c}{$95 \%$ C.I. for Exp(B) } \\
\cline { 7 - 10 } & & & & & & & & Lower & Upper \\
\hline Period & 0.056 & 0.002 & 728.449 & 1 & 0.000 & 1.057 & 1.053 & 1.061 \\
Life cycle & -0.046 & 0.001 & 4729.021 & 1 & 0.000 & 0.955 & 0.953 & 0.956 \\
Generation & 0.001 & 0.000 & 143.188 & 1 & 0.000 & 1.001 & 1.001 & 1.001 \\
Constant & -1.507 & 0.019 & 6601.033 & 1 & 0.000 & 0.221 & & \\
\hline
\end{tabular}

-2 Log likelihood $=48,406.071$.

Nagelkerke R2 $=0.197$. Predictive reliability $=69.4 \%$.

three effects (period, life cycle and generation) have a highly significant influence on the dependent variable. The period effect is the most powerful, followed by the life cycle effect and finally, the generation effect. This indicates that the period effects were the most important in the evolution of sporting practice in Spaniards between 1984 and 2014.

The life cycle effects have a negative sign while the period effects are positive and are shown to be stronger than those of the life cycle. The positive period effects cause an increase or prevent a decrease in the sports participation of diverse generations in a measure that compensates or surpasses the life cycle effect, a finding that has also been identified in Denmark (Pilgaard, 2013) and Germany (Breuer and Wicker, 2009).

The results of the logistic regression confirm the three hypotheses for which it was carried out, that is, $\mathrm{H}_{2}, \mathrm{H}_{3}$ and $\mathrm{H}_{4}$ and also suggest an additional confirmation to that which was previously obtained through the bivariate analysis. These results highlight the influence of the three effects on the evolution of sporting practice. It should also be noted that although the $\mathrm{R}^{2}$ and $\mathrm{B}$ coefficients had scores that were not overly high, this is normal in studies of sporting participation, thus it is more relevant to detain the analysis of statisti- cal significance level of the models (Scheerder and Vos, 2011). It should be noted that the three variables included in the model are not really good predictors of the total variance of the participation/no participation in the sporting activity (Pilgaard, 2013: 45).

\section{Conclusions}

This study has highlighted the fact that sporting practice in the distinct generational cohorts under study did not remain stable across the period, but rather, were subject to some significant variations. Therefore, $H_{1}$ was rejected, given that it proposed the stability of the sports participation patterns of the different generations based on the permanent influence of the attitudes and behaviors formed during the primary socialization period on the subsequent development (generation effect). This has been interpreted as proof of the fact that the period and cycle effects are stronger than the generation effects, a finding that comes as no surprise, given the great changes experienced in Spanish sports over the three analyzed decades - consideration of sports as a right of citizens, development of public sporting policies and an increase in the number of sports facilities (Llopis-Goig, 2016). 
Second, the analyses carried out have allowed us to corroborate $\mathrm{H}_{2}-$ a hypothesis also linked to the generation effect - given that it was possible to determine that with the passage of time, sporting practice carried out by individuals of a specific age increased with respect to the rate found for individuals of the same age at a distinct time. The multivariable analysis has corroborated this hypothesis, although its influence has been quantified to a lower degree than that found for period and life cycle effects. The bivariate analysis has confirmed this hypothesis for individuals born prior to the 1980s but not for those born in this decade and afterwards, given that this is the time when a turning point occurred in the evolution of sporting practice by virtue of which the youngest individuals no longer revealed increases in the same, as compared to individuals of their same age from earlier decades. This trend has already been seen in other European studies (Sheerder and Vos, 2011; Pilgaard, 2013) who explained this by the fact that the youngest cohorts had been socialized in contexts of greater pluralism, flexibility and orientation to change and had created less stable relationships with sporting activity (Twenge et al., 2010). The confirmation of this trend in the future would suggest a change in the evolution of sporting practice so as to imply a decrease in sporting activity in the youngest generations and, therefore, a certain separation of benefits provided by this active lifestyle. However, it should also be considered that the starting rates for these younger generations are higher than those for the older generations and therefore, the remaining question is whether or not sports socialization experienced at young ages has left sufficient mark, so as to influence future stages of their lives once they have surpassed the usual disengagement from organized sports that occurs during the adolescent phase (Coakley, 2015).

Third, the study has verified $H_{3}$, (life cycle effect) according to which as the individual's age increases, their sporting practice decreases given with the passage of the distinct phases of the life cycles changes take place that are related to the body, as well as modifications in the independence/dependence relationships that may affect the preferences or restrictions of the individual with regards to their leisure activities. However, a slight growth in the relationship between age and sports practice has been detected, which may be related to a greater awareness of individuals of a middle and advanced age about bodily care, and a greater commitment to a healthy and active lifestyle (Hirvensalo, Lampinen and Rantanen, 1998; Breuer and Wicker, 2009). This increased awareness and commitment to an active lifestyle would be the result of a period effect, which would have a greater influence on the older generations that are maintaining their physicalsporting activity rates after reaching their fifties and sixties.

Forth, the analyses have also confirmed $\mathrm{H}_{4}$. On the one hand, the logistics regression analysis has proven that the period effects were those having the greatest influence on the evolution of sporting practice in Spaniards between 1984 and $2014\left(H_{4}\right)$, followed by the life cycle effects $\left(H_{3}\right)$ and generation effects $\left(H_{2}\right)$. The generation effects have therefore been confirmed in line with that proposed in $\mathrm{H}_{2}$, but its impact has been lower than that of the life cycle $\left(H_{3}\right)$, which were in turn surpasses by those of period $\left(H_{4}\right)$. And this is why these latter (period effects) have consistently impacted all of the generational cohorts and at distinct times of the life cycle, highlighting that the combined impact of socio-cultural trends (lifestyles, culture of body and health) and public policies (sporting facilities, sports promotion programs) in the population may mean a challenge to the restrictions appearing with age and modify the interests and preferences that supposedly were produced during the primary socialization processes. The increase in plans, activities and facilities designed for physical 
activity in the elderly that were developed in the majority of the Spanish municipalities are especially relevant.

On the other hand, and regarding $H_{4}$, the bivariate analyses have provided a greater understanding of how period effects acted on the identification of the existence of three specific periods. The first took place between 1975 and 1984 and was related to the approval of the Constitution in 1978 as well as the entry into effect of Act 13/1980 Law on Physical Culture and Sports which resulted in the consideration of sports as a fundamental right of citizens as well as the increase in sporting facilities. The second took place between 1984 and 1995 and is explained by the extensive support and investment in sports occurring prior to the celebration of the 1992 Olympic Games in Barcelona, and by the sporting success that was achieved by the Spanish athletes at these games. The third period took place during the first decade of the $21^{\text {st }}$ century and was led by individuals of middle and advanced age who maintained their physical activity rates once reaching their forties, fifties and sixties, thanks to the influence of a new culture of health, wellbeing and quality of life.

\section{Bibliography}

Aiken, Leona S. and West, Stephen G. (1991). Multiple Regressions: Testing and Interpretation Interactions. Thousand Oaks, California: SAGE Publications.

Breedveld, Koen and Hoekman, Remco (2011). "Measuring Sports Participation in the Netherlands. The Need to Go Beyond Guidelines". European Journal for Sport and Society, 8(1/2): 117-132.

Breuer, Christoph (2005). "Cohort Effects in Physical Inactivity. A Neglected Category and Its Health Economical Implications". Journal of Public Health, 13(4): 189-195.

Breuer, Christoph and Wicker, Pamela (2009). "Decreasing Sports Activity with Increasing Age? Findings from a 20-year Longitudinal and Cohort
Sequence Analysis". Research Quarterly for Exercise and Sport, 80(1): 22-31.

Butcher, Janice; Lindner, Koenraad J. and Johns, David P. (2002). "Withdrawal from Competitive Youth Sport: A Retrospective Ten-year Study". Journal of Sport Behaviour, 25: 145-163.

Cea D’Ancona, María Á. (2002). Análisis multivariable. Teoría y práctica en la investigación social. Madrid: Síntesis.

Coakley, Jay (2015). Sports in Society. Issues and Controversies. New York: McGraw-Hill.

Engel, Claudia and Nagel, Siegfried (2011). "Sports Participation During the Life Course". European Journal for Sport and Society, 8(1/2): 45-63.

García-Ferrando, Manuel and Llopis-Goig, Ramón (2011). Ideal democrático y bienestar personal. Encuesta de Hábitos Deportivos en España 2010. Madrid: Centro de Investigaciones Sociológicas.

Gratton, Chris; Rowe, Nick and Veal, A. J. (2011). "International Comparisons of Sports Participation in European Countries: An Update of the COMPASS Project". European Journal for Sport and Society, 8(1/2): 99-116.

Hirvensalo, Mirja; Lampinen, Piiivi and Rantanen, Taina (1998). "Physical Exercise in Old Age. An Eight-year Follow-up Study on Involvement, Motives and Obstacles Among Persons Age 65-84". Journal of Aging and Physical Activity, 6: 157-168.

Ispizua, Marian and Monteagudo, María J. (2002). "Ocio y deporte: nuevas claves para el desarrollo humano". In: García Ferrando, M.; Lagardera, F. and Puig, N. (comps.). Sociología del deporte. Madrid: Alianza.

Jaime, Antonio M. (2008). "Trayectorias de participación política de la juventud europea: ¿Efectos de cohorte o efectos de ciclo vital?". Revista de Estudios de Juventud, 81: 67-93.

Klostermann, Claudia and Nagel, Siegfried (2014). "Changes in German Sport Participation: Historical Trends in Individual Sports". International Review for the Sociology of Sport, 49(5): 609634.

Llopis-Goig, Ramón (2016). "El sistema deportivo español. Estructura organizativa y pautas de participación ciudadana". In: Llopis-Goig, R. (ed.). La participación deportiva en Europa. Políticas, culturas y prácticas. Barcelona: UOC.

Martínez del Castillo, Jesús; Navarro, Carlos; Fraile, Antonio; Puig, Nuria; Jiménez, Pedro; Martínez, 
Javier and Miguel, Carlos de (1992). Deporte, sociedad y empleo. Madrid: Ministerio de Educación y Ciencia.

McPherson, Barry D.; Curtis, James E. and Loy, John W. (1993). The Social Significance of Sport. Champaign, Illinois: Human Kinetics.

Pilgaard, Maja (2013). "Age Specific Differences in Sports Participation in Denmark. Is Development Caused by Generation, Life Phase or Time Period Effects?". European Journal for Sport and Society, 10(1): 31-52.

Scheerder, Jeroen; Vanreusel, Bart and Taks, Marijke (2005). "Stratification Patterns of Active Sport Involvement Among Adults. Social Change and Persistence". International Review for the Sociology of Sport, 40(2): 139-162.

Scheerder, Jeroen and Vos, Steven (2011). "Social Stratification in Adult's Sports Participation from a Time-trend Perspective. Results from a 40-year
Household Study". European Journal for Sport and Society, 8(1/2): 31-44.

Scheerder, Jeroen; Vandermeerschen, Hanne; Van Tuyckom, Charlotte; Hoekman, Remco; Breedveld, Koen and Vos, Steven (2011). Understanding the Game: Sport Participation in Europe: Facts, Reflections and Recommendations. Available at: http://hdl.handle.net/1854/LU-1932490, access july 27, 2015.

Twenge, Jean M.; Campbell, Stacy M.; Hoffman, Brian J. and Lance, Charles E. (2010). "Generational Differences in Work Values: Leisure and Extrinsic Values Increasing, Social and Intrinsic Values Decreasing". Journal of Management, 36 (5): 1117-1142.

Yang, Yang (2011). "Aging, Cohorts and Methods". In: Binstock, R. H. and George, L. K. (eds). Handbook of Aging and the Social Sciences. Amsterdam: Elsevier.

RECEPTION: July 28, 2015

REVIEW: January 14, 2016

ACCEPTANCE: May 31, 2016 